\title{
Readily Available Phosphine-Phosphoramidite Ligands for Highly Efficient Rh-Catalyzed Enantioselective Hydrogenations
}

Jia-Di Huang, ${ }^{\dagger, \S}$ Xiang-Ping Hu, ${ }^{*, \dagger}$ Zheng-Chao Duan, ${ }^{\dagger, \S}$ Qing-Hen Zeng, ${ }^{\dagger, \S}$ Sai-Bo Yu, ${ }^{\dagger, \S}$ Jun Deng, ${ }^{\dagger, \S}$ Dao-Yong Wang ${ }^{\dagger, \S}$ and Zhuo Zheng ${ }^{*, \dagger}$

Dalian Institute of Chemical Physics, Chinese Academy of Sciences, 457 Zhongshan Road, Dalian 116023, China, and Graduate School of Chinese Academy of Sciences, Beijing 100039, China

Table of Contents

General Information

Synthesis of (S)-1-[2-(Diphenylphosphino)phenyl]ethylamine[(S)-DPPNH 3

Synthesis of (S)-N-Methyl-1-[2-(diphenylphosphino)phenyl]ethylamine[(S)-DPPNHMe] 4

General Procedure for the Synthesis of phosphine-phosphoramidite derivatives PEAPhos 1

General procedure for Asymmetric Hydrogenation

Determination of Enantiomeric Excesses for $\alpha$-amino acid esters 7a-e

Determination of Enantiomeric Excesses for $N$-Acetyl-1-Arylethylamine 9a-g

References

Copies of ${ }^{1} \mathrm{H},{ }^{13} \mathrm{C}$ and ${ }^{31} \mathrm{P}$ NMR Spectra for All New Compounds

Copies of Chromatographic Spectra of Hydrogenation Product 


\section{General Information}

All reactions were conducted under a nitrogen or argon atmosphere unless otherwise noted. Anhydrous procedures were conducted using oven dried or flame dried glassware and standard syringe and cannula transfer techniques. To eliminate the influence of environmental factors such as air and humidity in the catalytic reaction, hydrogenation reaction was carried out in glove-box by use of a stainless steel autoclave. Solvents were of reagent grade, dried and distilled before use following standard procedures. BINOL-based chlorophosphite 5 was synthesized according to the literature method ${ }^{1}$. $\alpha$-Dehydroamino acid esters $\mathbf{6 a -} \mathbf{e}^{2}$, and enamides $\mathbf{8} \mathbf{a}-\mathbf{g}^{3}$ were known compounds, which were synthesized according to the literature procedure. All other chemicals were obtained commercially. Optical rotations were recorded on a polarimeter at ambient temperature $(\mathrm{c}=\mathrm{g} / 100 \mathrm{~mL}) .{ }^{1} \mathrm{H},{ }^{13} \mathrm{C}$ and ${ }^{31} \mathrm{P}$ NMR spectra were recorded on a $400 \mathrm{MHz}$ instrument using $\mathrm{CDCl}_{3}$ as the solvent. Enantiomeric excesses were determined by capillary GC analysis with a chiral column.

\section{Synthesis of $(S)-1-\left[2-\left(\right.\right.$ diphenylphosphino)phenyl]ethylamine $\left[(S)-D^{-D P N H}{ }_{2}\right] 3$}

To a solution of $(S)$ - $\alpha$-phenylethylamine $2(1.21 \mathrm{~g}, 10.0 \mathrm{mmol})$ in $10 \mathrm{~mL}$ of ether at $-35{ }^{\circ} \mathrm{C}$ was dropwise added $4.0 \mathrm{~mL}(10.0 \mathrm{mmol})$ of a $2.5 \mathrm{M}$ solution of $n$-BuLi in hexanes. The resulting solution was stirred at $-35{ }^{\circ} \mathrm{C}$ for 15 minutes, and then $1.39 \mathrm{~mL}\left(11.0 \mathrm{mmol}, 1.1\right.$ equiv) of $\mathrm{Me}_{3} \mathrm{SiCl}$ was added slowly at the same temperature. The reaction mixture was stirred for 1 hour and then $12.0 \mathrm{~mL}(30.0$ mmol, 3 equiv) of a $2.5 \mathrm{M}$ solution of $n$-BuLi was added dropwise. After the addition was completed, the reaction mixture was stirred at $-35{ }^{\circ} \mathrm{C}$ for $3 \mathrm{~h}$. The reaction mixture was slowly warm to room temperature and stirred overnight. The reaction mixture was cooled to $-35^{\circ} \mathrm{C}$ again, and a solution of $1.80 \mathrm{~mL}(10.0 \mathrm{mmol})$ of chlorodiphenylphosphine in $10 \mathrm{~mL}$ of ether was added dropwise during 1 hour. The reaction mixture was stirred for another 3 hours at the same temperature, and then warmed to room temperature. After stirring for another 4 hours, a solution of $1 \mathrm{M}$ aqueous $\mathrm{HCl}$ was added slowly until the reaction mixture became clear in both phases. The aqueous phase was extracted with ether $(3 \times 10 \mathrm{~mL})$. Combined organic layers were dried over $\mathrm{Na}_{2} \mathrm{SO}_{4}$ and concentrated under reduced pressure. The residue was purified by silica gel column chromatography (hexanes / acetate, $8 / 1$ ) to give $1.22 \mathrm{~g}$ (40\% yield) of the targeted $(S)-\mathrm{DPPNH}_{2} 3$ as a white solid; mp 80 82 ${ }^{\circ} \mathrm{C} ;[\alpha]_{\mathrm{D}}{ }^{13}=-56.7\left(\mathrm{c} 0.53, \mathrm{CHCl}_{3}\right) ;{ }^{1} \mathrm{H}$ NMR $\left(\mathrm{CDCl}_{3}\right): \oint 1.23(\mathrm{~d}, J=6.8 \mathrm{~Hz}, 3 \mathrm{H}), 1.38(\mathrm{~s}, 2 \mathrm{H}), 4.90,(\mathrm{~m}, 1 \mathrm{H}), 6.83-7.59(\mathrm{~m}, 14 \mathrm{H}) ;{ }^{31} \mathrm{P} \mathrm{NMR}\left(\mathrm{CDCl}_{3}\right)$ : $\delta-16.3 ;{ }^{13} \mathrm{C}$ NMR $\delta 24.3,47.5,124.8,126.5,128.1,128.3,129.0,132.8,133.2,133.4,136.1,136.2$, 136.5, 136.6, 151.3, 151.5; HRMS (m/z) calcd for $\mathrm{C}_{20} \mathrm{H}_{20} \mathrm{NP}+\mathrm{H}: 306.1412$, found: 306.1406 . 


\section{Synthesis of $(S)$ - $N$-methyl-1-[2-(diphenylphosphino)phenyl]ethylamine[(S)-DPPNHMe] 4}

A mixture of $(S)-\mathrm{DPPNH}_{2} 3(1.22 \mathrm{~g}, 4 \mathrm{mmol})$ and ethyl formate $(1.45 \mathrm{~mL})$ was stirred at $45 \sim 50{ }^{\circ} \mathrm{C}$ overnight, and then volatile component was removed under reduced pressure to give the crude product, which did not need purification and used directly for next step.

In a $100 \mathrm{ml}$ freshly oven-dried three-necked flask placed $0.27 \mathrm{~g}$ of $\mathrm{LiAlH}_{4}$ and $10 \mathrm{~mL}$ of $\mathrm{THF}$ was slowly added a solution of above-mentioned crude product in $10 \mathrm{~mL}$ of THF under nitrogen atmosphere. The reaction mixture was refluxed for 5 hours, and then cooled to $0{ }^{\circ} \mathrm{C}$ by ice-bath. $10 \% \mathrm{KOH}$ aqueous solution $(5 \mathrm{~mL})$ was added slowly. The reaction mixture was filtered and the solid was thoroughly washed with THF. The solvent was removed under reduced pressure and the residue was purified by silica gel column chromatography (hexanes / acetate, $10 / 1)$ to give $0.66 \mathrm{~g}$ (52\% yield) of the targeted (S)-DPPNHMe 4 as a white solid; mp 81 83 ${ }^{\circ} \mathrm{C} ;[\alpha]_{\mathrm{D}}{ }^{23}=-57.1\left(\mathrm{c} 0.48, \mathrm{CHCl}_{3}\right) ;{ }^{1} \mathrm{H}$ NMR $\left(\mathrm{CDCl}_{3}\right): \delta$ 1.19-1.22 (d, $J=12 \mathrm{~Hz}, 3 \mathrm{H}), 2.14(\mathrm{~s}, 3 \mathrm{H}), 4.46-4.51$ (m, $1 \mathrm{H}), 6.84-7.53(\mathrm{~m}, 14 \mathrm{H}) ;{ }^{31} \mathrm{P} \mathrm{NMR}\left(\mathrm{CDCl}_{3}\right): \delta$ $16.5 ;{ }^{13} \mathrm{C}$ NMR $\delta 23.2,34.2,56.6,125.8,125.9,126.9,128.5,128.6,128.7,129.4,133.4,133.8,134.0$, 134.2, 135.2, 136.7, 136.8, 137.0, 137.1, 149.8, 150.0; HRMS (m/z) calcd for $\mathrm{C}_{21} \mathrm{H}_{22} \mathrm{NP}: 319.1490$, found: 319.1492 .

\section{General Procedure for the Synthesis of phosphine-phosphoramidite derivatives PEAPhos 1}

$(R)$-Chlorophosphite $5(350.5 \mathrm{mg}, 1.0 \mathrm{mmol})$ was dissolved in $4.0 \mathrm{~mL}$ of dried toluene, which was cooled to $0{ }^{\circ} \mathrm{C}$. A solution of $(S)$-DPPNH 3 or $(S)$-DPPNHMe 4 (1.0 mmol) and $\mathrm{Et}_{3} \mathrm{~N}$ (303 mg, 3.0 $\mathrm{mmol}$ ) in $4.0 \mathrm{~mL}$ of toluene was added to above solution during $30 \mathrm{~min}$. The resulting mixture was left standing at room temperature overnight. The precipitate was filtered, and the solid was washed with toluene $(5 \mathrm{~mL} \times 1)$. The filtrate was collected, and concentrated under reduced pressure. The residue was purified by column chromatography to give the crude product, which can be further purified by crystallizing from hexane/dichloromethane.

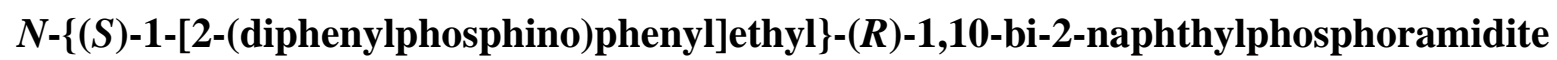

$\left(S_{c}, R_{a}\right)-$ PEAPhos 1a: white solids; mp 107 109 ${ }^{\circ} \mathrm{C} ;[\alpha]_{\mathrm{D}}{ }^{24}=-87.0\left(\mathrm{c} 1.02, \mathrm{CHCl}_{3}\right) ;{ }^{1} \mathrm{H} \mathrm{NMR}\left(\mathrm{CDCl}_{3}\right): \delta 1.33-$ $1.35(\mathrm{~d}, J=6.8 \mathrm{~Hz}, 3 \mathrm{H}), 3.68-3.75(\mathrm{~m}, 1 \mathrm{H}), 5.37-5.45(\mathrm{~m}, 1 \mathrm{H}), 6.71-7.92(\mathrm{~m}, 26 \mathrm{H}) ;{ }^{31} \mathrm{P} \mathrm{NMR}\left(\mathrm{CDCl}_{3}\right) ; \delta$ -18.0, 152.7; ${ }^{13} \mathrm{C}$ NMR $\delta 25.6,48.4,122.5,124.7,125.9,126.0,126.9,127.0,128.2,128.3,128.5,128.6$, $128.7,129.6,133.6,133.8,133.9,134.0,134.1,136.8,147.4,149.4,150.8 ;$ HRMS (m/z) calcd for $\mathrm{C}_{40} \mathrm{H}_{31} \mathrm{NO}_{2} \mathrm{P}_{2}$ : 619.1830, found: 619.1835 . 


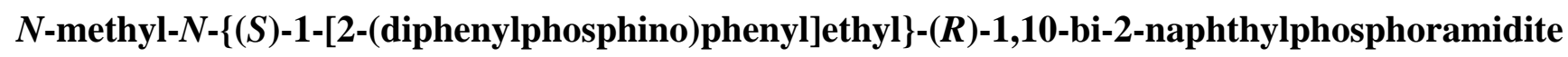
$\left(\boldsymbol{S}_{\boldsymbol{c}}, \boldsymbol{R}_{\boldsymbol{a}}\right)$-PEAPhos 1b: white solids; mp $174 \sim 176{ }^{\circ} \mathrm{C} ;[\alpha]_{\mathrm{D}}{ }^{24}=-122.9\left(\mathrm{c} 1.01, \mathrm{CHCl}_{3}\right) ;{ }^{1} \mathrm{H} \mathrm{NMR}\left(\mathrm{CDCl}_{3}\right)$ : $\delta 1.60-1.62(\mathrm{~d}, J=9.88 \mathrm{~Hz}, 3 \mathrm{H}), 1.99(\mathrm{~s}, 3 \mathrm{H}), 5.40-5.44(\mathrm{~m}, 1 \mathrm{H}), 7.03-7.94(\mathrm{~m}, 26 \mathrm{H}) ;{ }^{31} \mathrm{P}$ NMR $\left(\mathrm{CDCl}_{3}\right): \delta-18.4,148.4 ;{ }^{13} \mathrm{C} \mathrm{NMR} \delta 21.6,30.5,56.6,122.1,124.4,125.96,125.99,126.9,127.0,128.1$, $128.3,128.5,128.6,128.7,129.3,129.9,130.1$, 133.7, 133.9, 134.0, 136.9, 147.8, 149.8, 150.5; HRMS (m/z) calcd for $\mathrm{C}_{41} \mathrm{H}_{33} \mathrm{NO}_{2} \mathrm{P}_{2}: 633.1987$, found: 633.1981 .

\section{$N$-\{(S)-1-[2-(diphenylphosphino)phenyl]ethyl\}-(S)-1,10-bi-2-naphthylphosphoramidite}

$\left(S_{c}, S_{a}\right)-$

PEAPhos 1c: white solids; mp $108 \sim 110{ }^{\circ} \mathrm{C} ;[\alpha]_{\mathrm{D}}{ }^{24}=56.5\left(\mathrm{c} 0.44, \mathrm{CHCl}_{3}\right) ;{ }^{1} \mathrm{H} \mathrm{NMR}\left(\mathrm{CDCl}_{3}\right): \delta 1.22-$ $1.26(\mathrm{~d}, J=14 \mathrm{~Hz}, 3 \mathrm{H}), 3.46-3.49(\mathrm{~m}, 1 \mathrm{H}), 5.44(\mathrm{~m}, 1 \mathrm{H}), 6.92-7.92(\mathrm{~m}, 26 \mathrm{H}) ;{ }^{31} \mathrm{P} \mathrm{NMR}\left(\mathrm{CDCl}_{3}\right): \delta-$ 16.9, 153.9; ${ }^{13} \mathrm{C}$ NMR $\delta 26.0,48.5,122.6,124.7,126.0,126.9,127.0,128.2,128.3,128.5,128.6,128.7$, 128.8, 129.6, 133.7, 133.92, 134.0, 134.1, 147.2, 149.4, 150.3; HRMS (m/z) calcd for $\mathrm{C}_{40} \mathrm{H}_{31} \mathrm{NO}_{2} \mathrm{P}_{2}$ : 619.1830, found: 619.1825 .

\section{$N$-methyl- $N$ - $\{(S)-1-[2-($ diphenylphosphino)phenyl]ethyl\}-(S)-1,10-bi-2-naphthylphosphoramidite} $\left(\boldsymbol{S}_{\boldsymbol{c}}, \boldsymbol{S}_{\boldsymbol{a}}\right)$-PEAPhos 1d: white solids; mp 206 208 ${ }^{\circ} \mathrm{C} ;[\alpha]_{\mathrm{D}}{ }^{24}=195.7\left(\mathrm{c} 0.58, \mathrm{CHCl}_{3}\right) ;{ }^{1} \mathrm{H}$ NMR $\left(\mathrm{CDCl}_{3}\right)$ : $\delta 1.66-1.69(\mathrm{~d}, J=9.8 \mathrm{~Hz}, 3 \mathrm{H}), 1.87(\mathrm{~s}, 3 \mathrm{H}), 5.42(\mathrm{~m}, 1 \mathrm{H}), 6.99-7.91(\mathrm{~m}, 26 \mathrm{H}) ;{ }^{31} \mathrm{P} \mathrm{NMR}\left(\mathrm{CDCl}_{3}\right): \delta-$ 17.8, 147.6; ${ }^{13} \mathrm{C}$ NMR $\delta 21.6,30.1,56.7,124.4,125.9,127.00,127.06,127.5,128.2,128.3,128.4,128.5$, 128.6, 128.7, 130.1, 133.7, 133.9, 134.0, 147.4, 149.7, 150.7; HRMS (m/z) calcd for $\mathrm{C}_{41} \mathrm{H}_{33} \mathrm{NO}_{2} \mathrm{P}_{2}$ : 633.1987, found: 633.1992 .

\section{General procedure for asymmetric hydrogenation}

In a nitrogen-filled glovebox, a stainless steel autoclave was charged with $\mathrm{Rh}(\mathrm{COD})_{2} \mathrm{BF}_{4}(2.0 \mathrm{mg}, 0.5 \mathrm{x}$ $\left.10^{-2} \mathrm{mmol}\right)$ and PEAPhos $1\left(0.55 \times 10^{-2} \mathrm{mmol}\right)$ in $1.5 \mathrm{~mL}$ of a degassed $\mathrm{CH}_{2} \mathrm{Cl}_{2}$. After stirring for 10 min at room temperature. A substrate $(0.5 \mathrm{mmol})$ in $1.5 \mathrm{~mL}$ of the same solvents was added to the reaction mixture, and then the hydrogenation was performed at room temperature under an $\mathrm{H}_{2}$ pressure of 10 bar for 12 hours. The reaction mixture was passed through a short silica gel column to remove the catalyst. After evaporating the solvent, the crude reaction mixture was subjected to GC to determine the enantiomeric excesses and yields of hydrogenation products.

\section{Determination of Enantiomeric Excesses for $\alpha$-amino acid esters 7a-e:}


Chiral Capillary GC Column. CP-Chiralsil-L-Val column (25m x $0.25 \mathrm{~mm}$ x $0.12 \mu \mathrm{m})$. Carrier gas: $\mathrm{N}_{2}$. The racemic products were obtained by hydrogenation of substrates with an achiral catalyst prepared from $\mathrm{PPh}_{3}$ and $\mathrm{Rh}(\mathrm{COD})_{2} \mathrm{BF}_{4}$. The following are the retention times for the racemic products.

Methyl 2-Acetamido-3-phenylpropanoate (7a): (capillary GC, CP-Chiralsil-L-Val column, $160{ }^{\circ} \mathrm{C}, 20$ psi) $\mathrm{t}(\mathrm{R})=8.59, \mathrm{t}(\mathrm{S})=9.45$.

Methyl 2-Acetamido-3-(2-chlorophenyl)propanoate (7b): (capillary GC, CP-Chiralsil-L-Val column, $\left.160{ }^{\circ} \mathrm{C}, 20 \mathrm{psi}\right) \mathrm{t}(\mathrm{R})=16.45, \mathrm{t}(\mathrm{S})=18.32$.

Methyl 2-Acetamido-3-(4-chlorophenyl)propanoate (7c): (capillary GC, CP-Chiralsil-L-Val column, $\left.160{ }^{\circ} \mathrm{C}, 20 \mathrm{psi}\right) \mathrm{t}(\mathrm{R})=22.67, \mathrm{t}(\mathrm{S})=25.95$.

Methyl 2-Acetamido-3-(2-methoxyphenyl)propanoate (7d): (capillary GC, CP-Chiralsil-L-Val column, $\left.160{ }^{\circ} \mathrm{C}, 20 \mathrm{psi}\right) \mathrm{t}(\mathrm{R})=16.79, \mathrm{t}(\mathrm{S})=18.54$.

Methyl 2-Acetamido-3-(4-methoxyphenyl)propanoate (7e): (capillary GC, CP-Chiralsil-LValcolumn, $\left.160^{\circ} \mathrm{C}, 20 \mathrm{psi}\right) \mathrm{t}(\mathrm{R})=23.19, \mathrm{t}(\mathrm{S})=25.81$.

Determination of Enantiomeric Excesses for $\boldsymbol{N}$-Acetyl-1-Arylethylamine 9a-g:

Chiral Capillary GC Column. Chiral Select-1000 column (dimensions 30 m x 0.25 mm(i.d.)). Carrier gas: $\mathrm{N}_{2}$. The racemic products were obtained by hydrogenation of substrates with an achiral catalyst prepared from $\mathrm{PPh}_{3}$ and $\mathrm{Rh}(\mathrm{COD})_{2} \mathrm{BF}_{4}$. The following are the retention times for the racemic products.

$N$-Acetyl-1-phenylethylamine (9a): (capillary GC, Chiral Select-1000 column, $\left.130{ }^{\circ} \mathrm{C}, 10 \mathrm{psi}\right) \mathrm{t}(S)=$ $21.44, \mathrm{t}(R)=23.38$.

$N$-Acetyl-1-(4-methylphenyl)ethylamine (9b): (capillary GC, Chiral Select-1000 column, $130{ }^{\circ} \mathrm{C}, 15$ psi) $\mathrm{t}(S)=25.12, \mathrm{t}(R)=26.97$.

$N$-Acetyl-1-(4-trifluoromethyl)ethylamine (9c): (capillary GC, Chiral Select-1000 column, $130{ }^{\circ} \mathrm{C}, 15$ psi) $\mathrm{t}(S)=22.70, \mathrm{t}(R)=25.06$.

$N$-Acetyl-1-(4-bromophenyl)ethylamine (9d): (capillary GC, Chiral Select-1000 column, $140{ }^{\circ} \mathrm{C}, 15$ psi) $\mathrm{t}(S)=62.43, \mathrm{t}(R)=66.18$.

$\mathrm{N}$-Acetyl-1-(4-chlorophenyl)ethylamine (9e): (capillary GC, Chiral Select-1000 column, $140{ }^{\circ} \mathrm{C}, 10$ psi) $\mathrm{t}(S)=51.15, \mathrm{t}(R)=54.35$.

$N$-Acetyl-1-(4-methoxyphenyl)ethylamine (9f): (capillary GC, Chiral Select-1000 column, $140{ }^{\circ} \mathrm{C}, 10$ psi) $\mathrm{t}(S)=58.02, \mathrm{t}(R)=60.98$.

$N$-Acetyl-1-(3-methoxyphenyl)ethylamine (9g): (capillary GC, Chiral Select-1000 column, $130{ }^{\circ} \mathrm{C}$, 10psi) $\mathrm{t}(S)=78.30, \mathrm{t}(R)=83.16$. 


\section{References and Notes}

1. Franciò, G.; Arena, C. G.; Faraone, F.; Graiff, C.; Lanfranchi, M.; Tiripicchio, A. Eur.J. Inorg. Chem. 1999, 1219.

2. Blott, A. H. Org. Syn., Coll. Vol. 1950, p1

3. (a) van den Berg, M.; Minnaard, A. J.; Schudde, E. P.; van Esch, J.; de Vries, J. G.; Feringa, B. L. J. Am. Chem. Soc. 2000, 122, 11539. (b) Burk, M. J.; Casy, G.; Johnson, N. B. J. Org. Chem. 1998, 63, 6084 . 
1H NMR HJD-1 IN CDCL3 D4/11/22
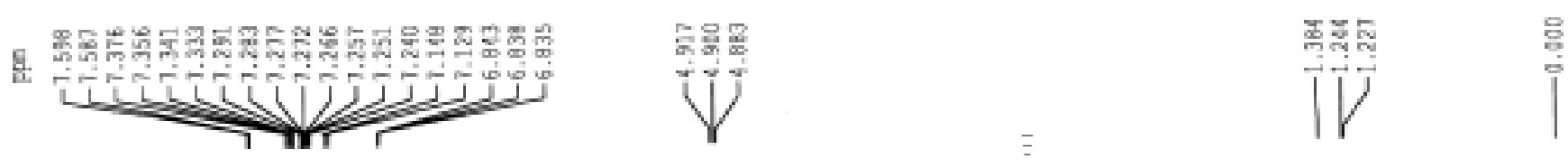

$$
\mathrm{NH}_{2}
$$

(S)-DPPNH 3

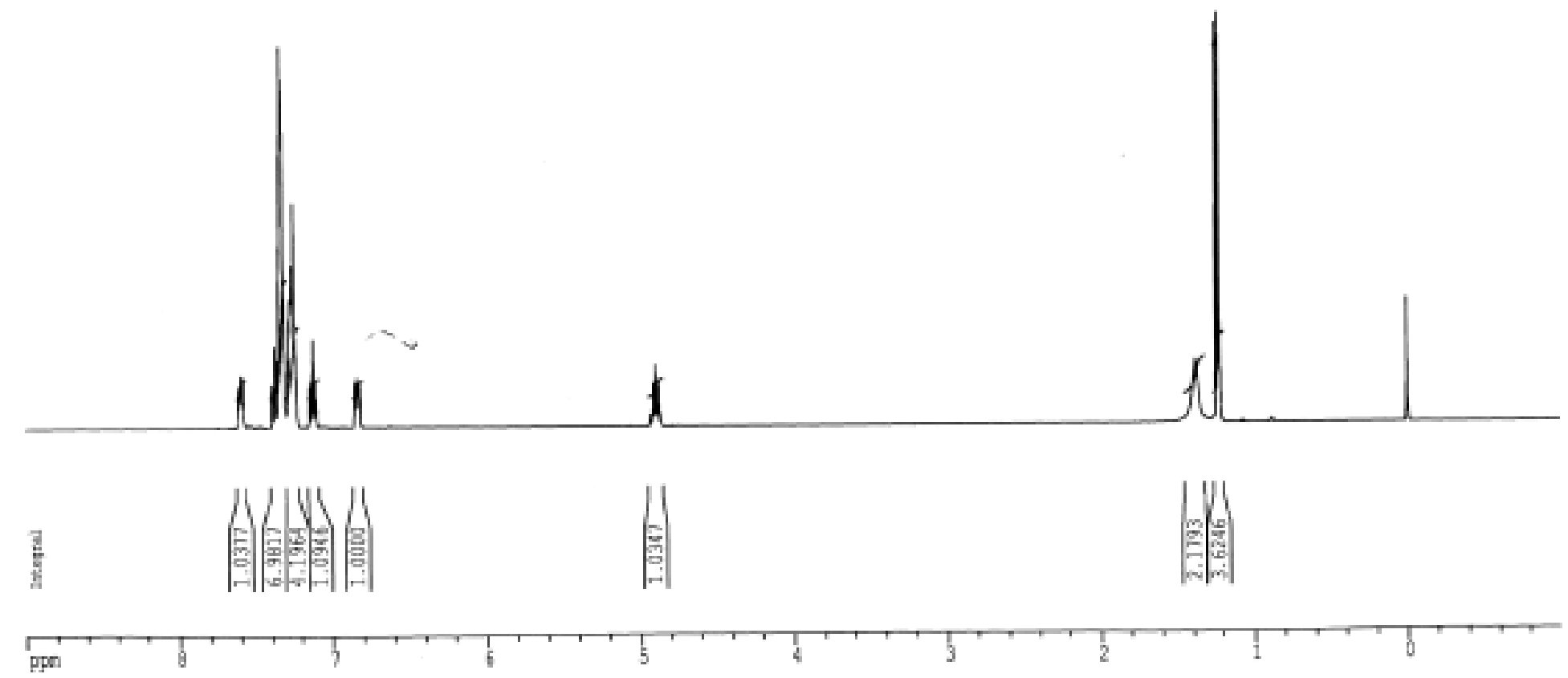


(S)-DPPNH 3

(1)

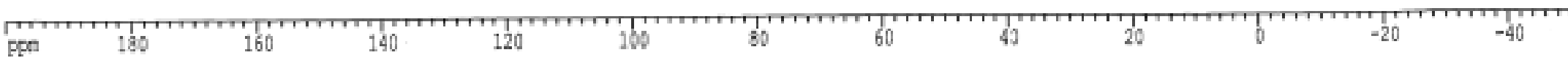




$$
\text { . }
$$


1H NMB HJD-1 IN CDCL 3 06/06/13

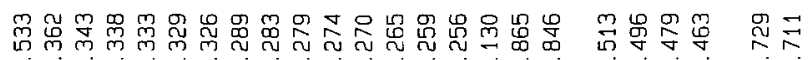

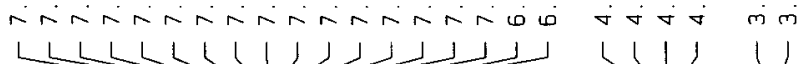
(1)

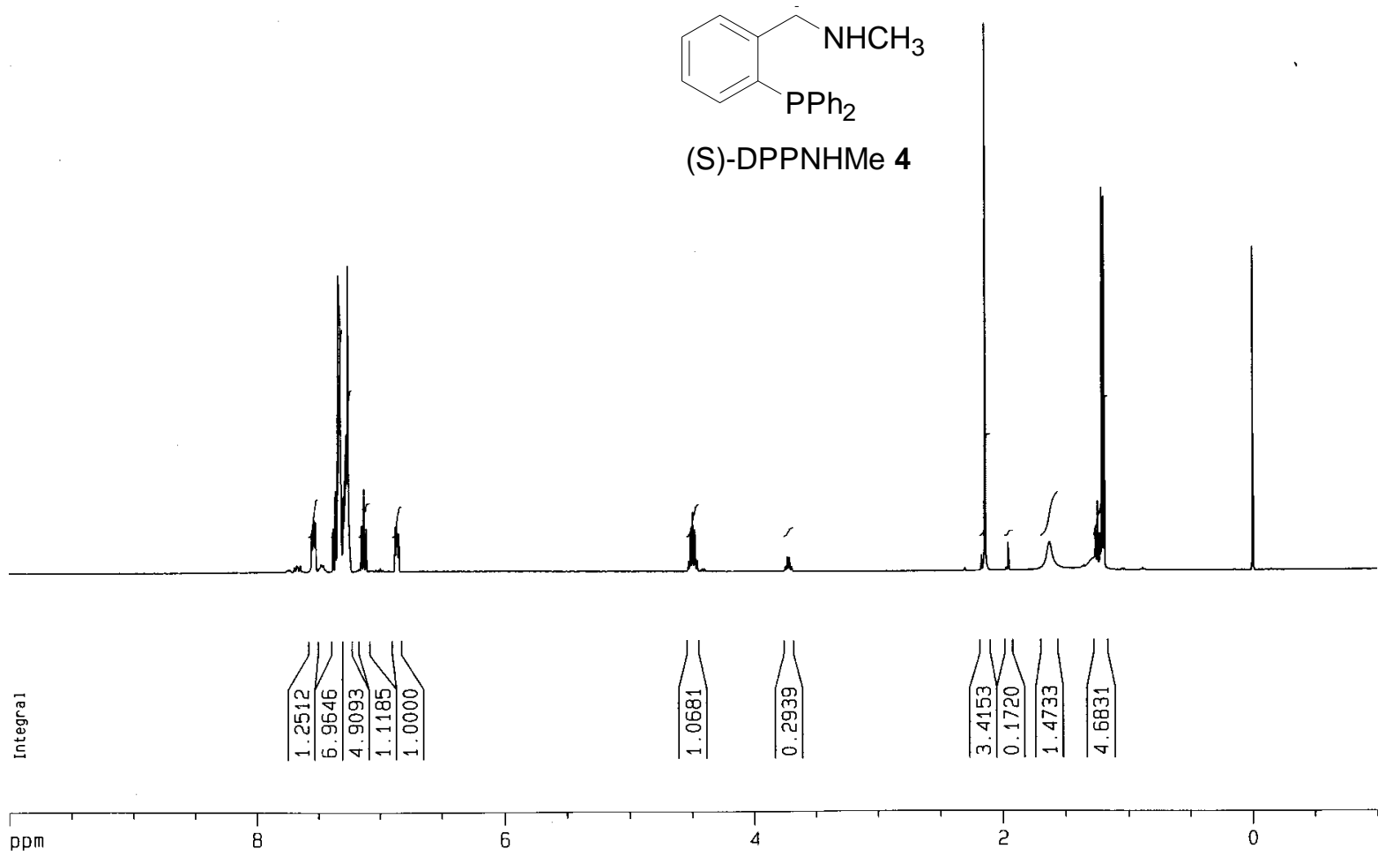

Current Data Parameters

NAME

PROCNO

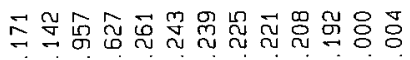

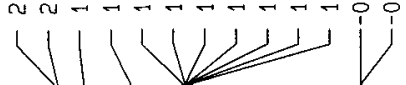

Date-

Time

14.23
ar

PUIPROC

SOLVENT

NS

DS

IDRES $\quad 0.244532 \mathrm{~Hz}$

AQ $\quad 2.0447731 \mathrm{sec}$

$\begin{array}{lr}\text { RG } & 181 \\ \text { DW } & 62.400 \text { usec }\end{array}$

DE $\quad 6.00 \mathrm{usec}$

1.00000000

MCREST $\quad 0.00000000$ Sec

MCWRK $\quad 0.01500000 \mathrm{sec}$

$=z:====$ CHANNEL $f 1$ $=z== \pm==$

$\begin{array}{ll}\text { NUC1 } & 1 \mathrm{H} \\ \mathrm{O} 1 & 8.20 \mathrm{use}\end{array}$

P1 8.20 usec

SF01 400.1332521 MHz

F2 - Processing parameters

SI 32768

WDW $\quad 400.1300177$ MrE

$\begin{array}{cc}\text { WOW } & \text { GM } \\ \text { SSB } & 0 \\ \text { L } & -0.50 \mathrm{~Hz}\end{array}$

$\begin{array}{lr}L B & -0.50 \\ G B & 0.1 \\ \text { PC } & 1.40\end{array}$

10 NMR plot parameters

CX $20.00 \mathrm{~cm}$

$\begin{array}{lr}\text { CY } & 8.00 \mathrm{~cm} \\ \text { FIP } & 10.000 \mathrm{pmm}\end{array}$

$10.000 \mathrm{DDm}$

$\begin{array}{ll}F 1 & 4001.30 \mathrm{~Hz}\end{array}$

F2 $-1.000 \mathrm{pp}$

PPMCM $\quad 0.55000 \mathrm{ppm} / \mathrm{cm}$ 
31P NMR HJD-1 IN CDCL3 06/06/13

言

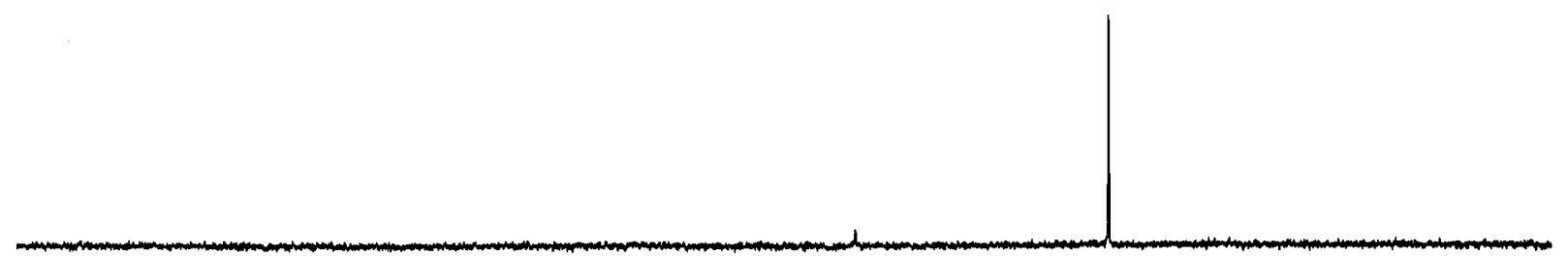

$\stackrel{6}{\circ}$

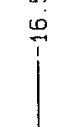

$\mathrm{PPh}_{2}$

(S)-DPPNHMe 4

150

100

50

Current Data Parameters
NAME
hid
EXPNO

EXPNO
PROCNO

F2 - Acquisition Parameters

Date_ 20060613

$\begin{array}{ll}\text { Time } & 14.19 \\ \text { INSTRUM } & \text { ar } \times 400\end{array}$

PROBHD $5 \mathrm{~mm}$ PABBO BE-

$\begin{array}{lr}\text { PULPROG } & 29 \\ \text { ID } & 32768 \\ \text { SOLVENT } & \text { COC13 } \\ \text { NS } & 117 \\ \text { DS } & 11\end{array}$

DS
SWH $\quad 48543.688 \mathrm{~Hz}$

SWH
FIDRES

AQ $\quad 0.3375604 \mathrm{sec}$

RG $\quad 3251$

$\begin{array}{lr}\text { DW } & 10.300 \text { usec } \\ \text { DE } & 6.00 \text { usec } \\ \text { TE } & 297.2 \mathrm{~K}\end{array}$

$\begin{array}{ll}\text { TE } & 297.2 \mathrm{~K} \\ 01 & 200000000\end{array}$

$\begin{array}{ll}\text { MCREST } & 2.00000000 \mathrm{sec} \\ \text { MCWFK } & 0.00000000 \mathrm{sec}\end{array}$

$\begin{array}{ll}\text { MCWEST } & 0.00000000 \mathrm{sec} \\ \text { MCWFK } & 0.01500000 \mathrm{sec}\end{array}$

$=======$ CHANNEL $+1=======$
NUC1 $31 \mathrm{P}$

$\begin{array}{ll}\text { NUC1 } & 31 P \\ \text { P1 } & 8.00 \text { usec } \\ \text { PL1 } & 0.00 \text { di }\end{array}$

$\begin{array}{lr}\text { PL1 } & 0.00 \mathrm{~dB} \\ \text { SFO1 } & 161.9820520 \mathrm{MHZ}\end{array}$

F2 - Processing parameters

SI 1610.07534

SF $\quad 161.9755337 \mathrm{MHZ}$

SLB

$\stackrel{L B}{G B}$

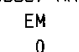

0
1.40

10 NMR plot parameters

$C X$
$C Y$$\quad 20.00 \mathrm{~cm}$

$\begin{array}{ll}C Y & 3.00 \mathrm{~cm} \\ F 1 P & 190.000 \mathrm{ppm}\end{array}$

$\begin{array}{ll}F_{1} & 30775.35 \mathrm{~Hz} \\ F 2 P & -100.000 \mathrm{ppm}\end{array}$

F2 $\quad-16197.55 \mathrm{~Hz}$

$\begin{array}{lr}\text { PPMCM } & 14.50000 \mathrm{ppm} / \mathrm{cm} \\ \mathrm{HZCM} & 2348.64526 \mathrm{~Hz} / \mathrm{cm}\end{array}$ 
13C NMR HJD-2 IN CDCL3 06/06/13

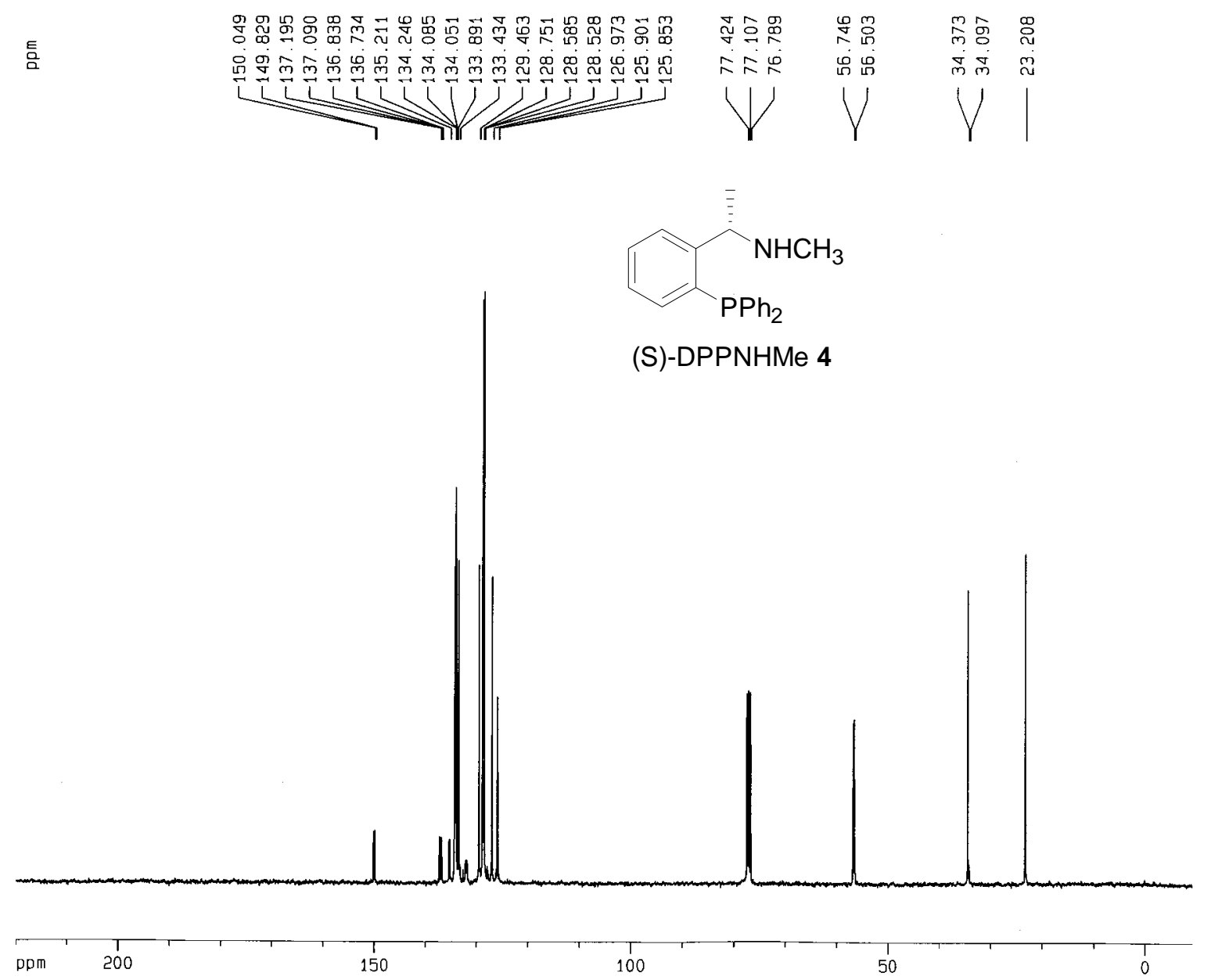

Current Data Parameters

$\begin{array}{ll}\text { NAME } & \text { hjd } \\ \text { EXPNO } & 199 \\ & \end{array}$

F2 - Acquisition Parameters

Date 20060613

Time

$\mathrm{dr} \times 400$

PULPROG $5 \mathrm{~mm}$ PABBO

$\begin{array}{ll}\text { TD } & 65536 \\ \text { SOLVENT } & \text { COC13 }\end{array}$

$\begin{array}{lr}\text { NS } & 690 \\ \text { OS } & 16\end{array}$

$\begin{array}{lr}23148.148 \mathrm{~Hz} \\ \text { SWH } & 0353213 \mathrm{~Hz}\end{array}$

$\begin{array}{ll}\text { FIDRES } & 0.353213 \mathrm{~Hz} \\ \mathrm{AQ} & 1.4156276 \mathrm{sec}\end{array}$

$\begin{array}{lr}\text { RG } & 16384 \\ \text { DW } & 21.600 \text { use }\end{array}$

$6.00 \mathrm{use}$
$\mathrm{DE}$

$01 \quad 1.00000000 \mathrm{sec}$

DCREST $\quad 0.03000000 \mathrm{sec}$

MCWFK $\quad 0.00000000 \mathrm{sec}$

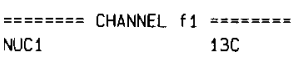

$\begin{array}{ll}\text { P1 } & \text { B. } 20 \text { use } \\ \text { PL1 } & 1.50 \mathrm{~dB}\end{array}$

$=======$ CHANNEL $f 2== \pm==3$

CPDPRG2
waltz16

$\begin{array}{ll}\text { NUC2 } & 1 \mathrm{H} \\ \text { PCPD2 } & 80.00\end{array}$

PL2 $5.00 \mathrm{~dB}$

SFO2 $\quad \begin{array}{rl}23.00 & \mathrm{OB} \\ \mathrm{OHz} & 400.1320007 \mathrm{MHz}\end{array}$

$\begin{array}{lr}\text { F2 - Pracessing parameters } \\ \text { SI } & 32768 \\ \text { SF } & 100.6127690 \\ \text { MHZ }\end{array}$

WOW $\quad 100.6127690 \mathrm{MH}$

$\begin{array}{lc}\text { SSB } & 0 \\ \mathrm{LB} & 4.00 \mathrm{~Hz} \\ \mathrm{~GB} & 0 \\ \triangle \mathrm{C} & 1.40\end{array}$

10 NMA plot parameters

$\begin{array}{ll}\text { CX } \\ C Y & 20.00 \mathrm{~cm} \\ C y & \end{array}$

$\begin{array}{lr}\text { CY } & 10.00 \mathrm{~cm} \\ F 1 \mathrm{P} & 220.000 \mathrm{ppm} \\ \varepsilon_{1} & 22130.81 \mathrm{~Hz}\end{array}$

$\begin{array}{ll}F 1 & 22134.81 \mathrm{~Hz} \\ \text { F } 2 \mathrm{P} & -10.000 \mathrm{ppm}\end{array}$

$\begin{array}{ll}\text { PPMCM } & -1006.13 \mathrm{~Hz} \\ & 11.50000 \mathrm{ppm} / \mathrm{c}\end{array}$ 
1H КМ̈ H.O-1 IN CDCL 3 06/05/30

\section{8}

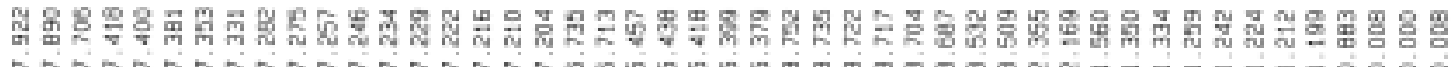
in

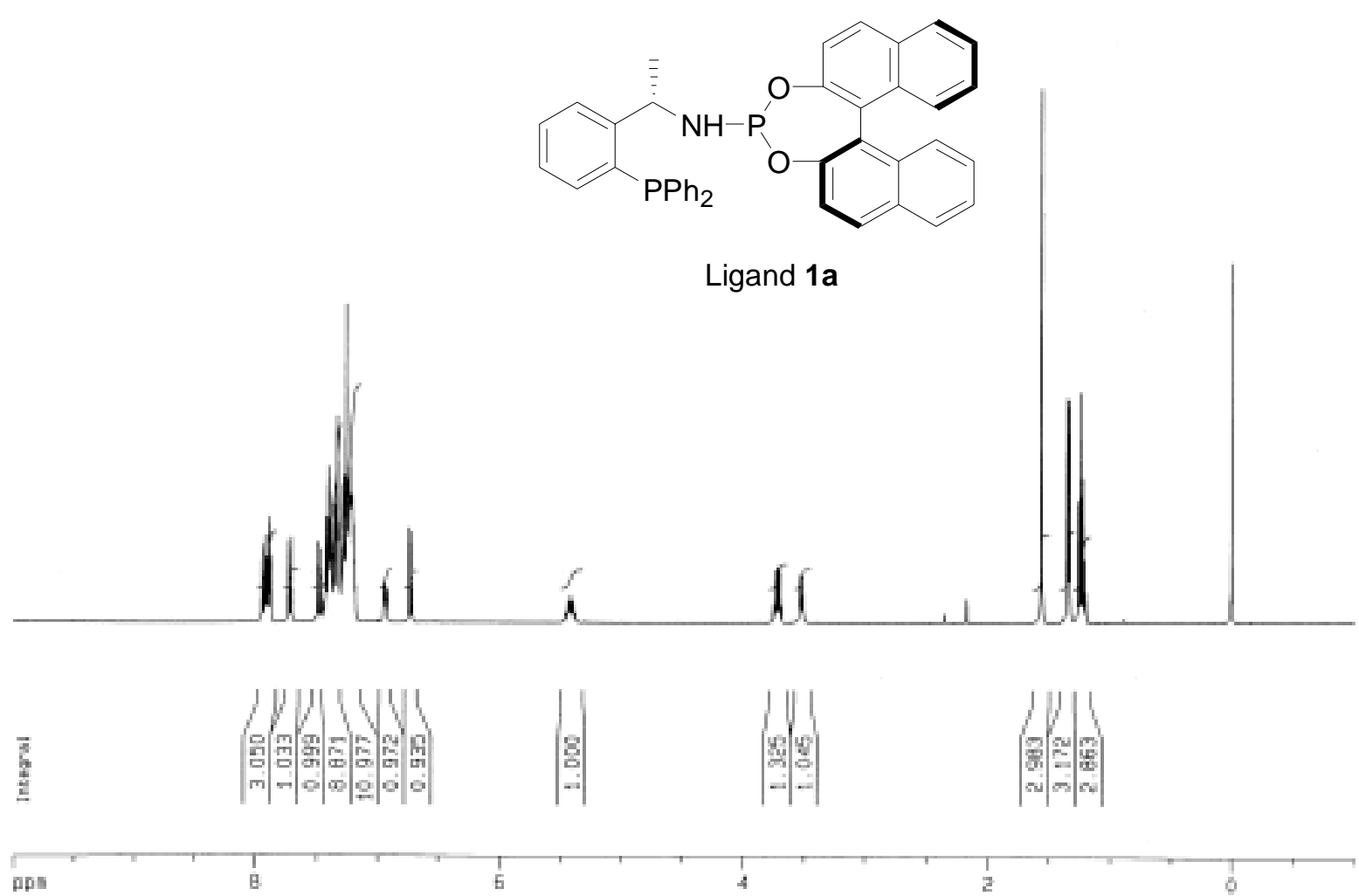

wat $A$ he

12 - Atquisutian Paraneters

bute_ 20060530

Tine

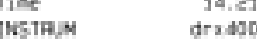

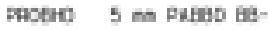

The

50. vent $\quad \operatorname{coc} 13$

15

Swer S012.0220 $\mathrm{Hz}$

$0.246532 \mathrm{~Hz}$
Fibes

48 कृ

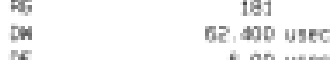

TE 5.00 uie

ot $1.0000000 \mathrm{sec}$

acrest $\quad 0.00000000$ sec

$0.0030000 \mathrm{set}$

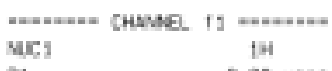

5. 5.20 uate

sFos $\quad 400 \quad 5.06$ da

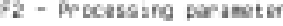

51 A 12765

gF $\quad 400.1300195 \mathrm{kes}$

558

$5 S H$
GB
$G B$

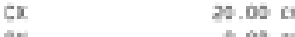

$\begin{array}{ll}\text { CY } & 5.05 \mathrm{~cm} \\ \text { E. } & 10.009 \mathrm{~cm}\end{array}$

F1 a0s. $30 \mathrm{~Hz}$

con

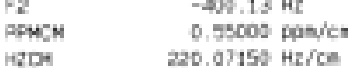


31P Rer H.JD-1 [N $\operatorname{coc} 306 / 05 / 30$

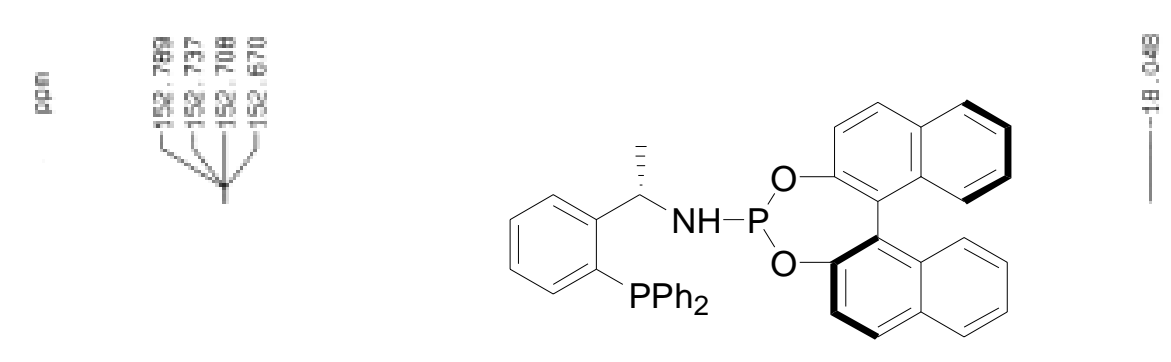

Ligand 1a

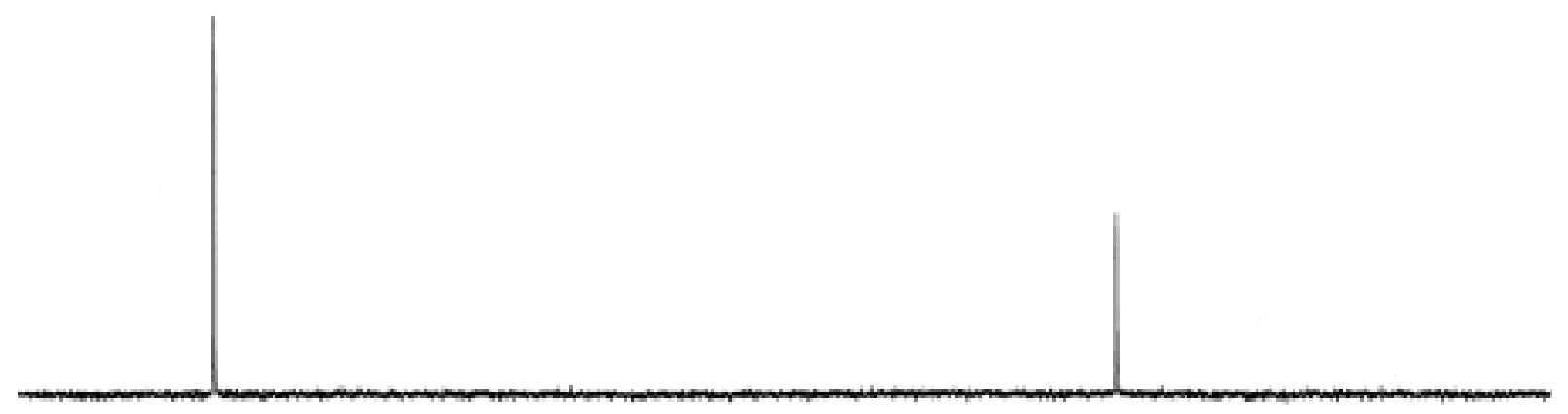

ppn

150

100

50

0 $\ln _{-50}^{1}$

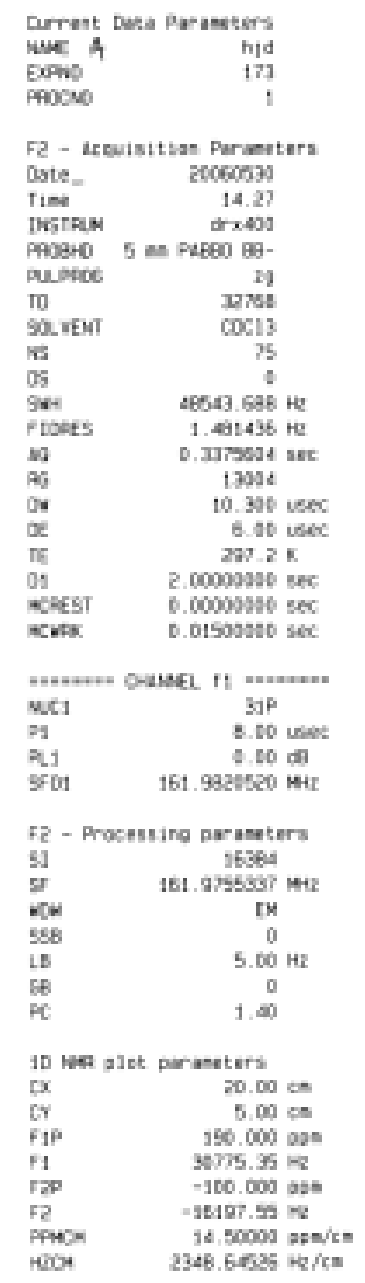

carrent Desal Paraseter-

Fe - Lrouisttian Parmeter

Ec06053

tonem

05

Sort $46643.698 \mathrm{rog}$

tobes 1. $4.6936 \mathrm{~kg}$

13094

$10.330 \mathrm{vec}$
$6.30 \mathrm{usec}$

copsose sec

. $00000350 \mathrm{sec}$

to nies

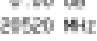

8- Procensing peraseters

51 161.97555357 nets

Cow $5 \mathrm{CM}$

$\begin{array}{ll}15 & 5.00 \mathrm{~Hz} \\ \mathrm{LE} & 1.00\end{array}$

id wee alet paraneters

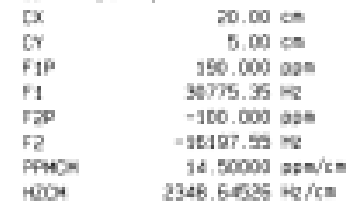


13C. NeP N.0-2 IN CDCL3 06/06/05

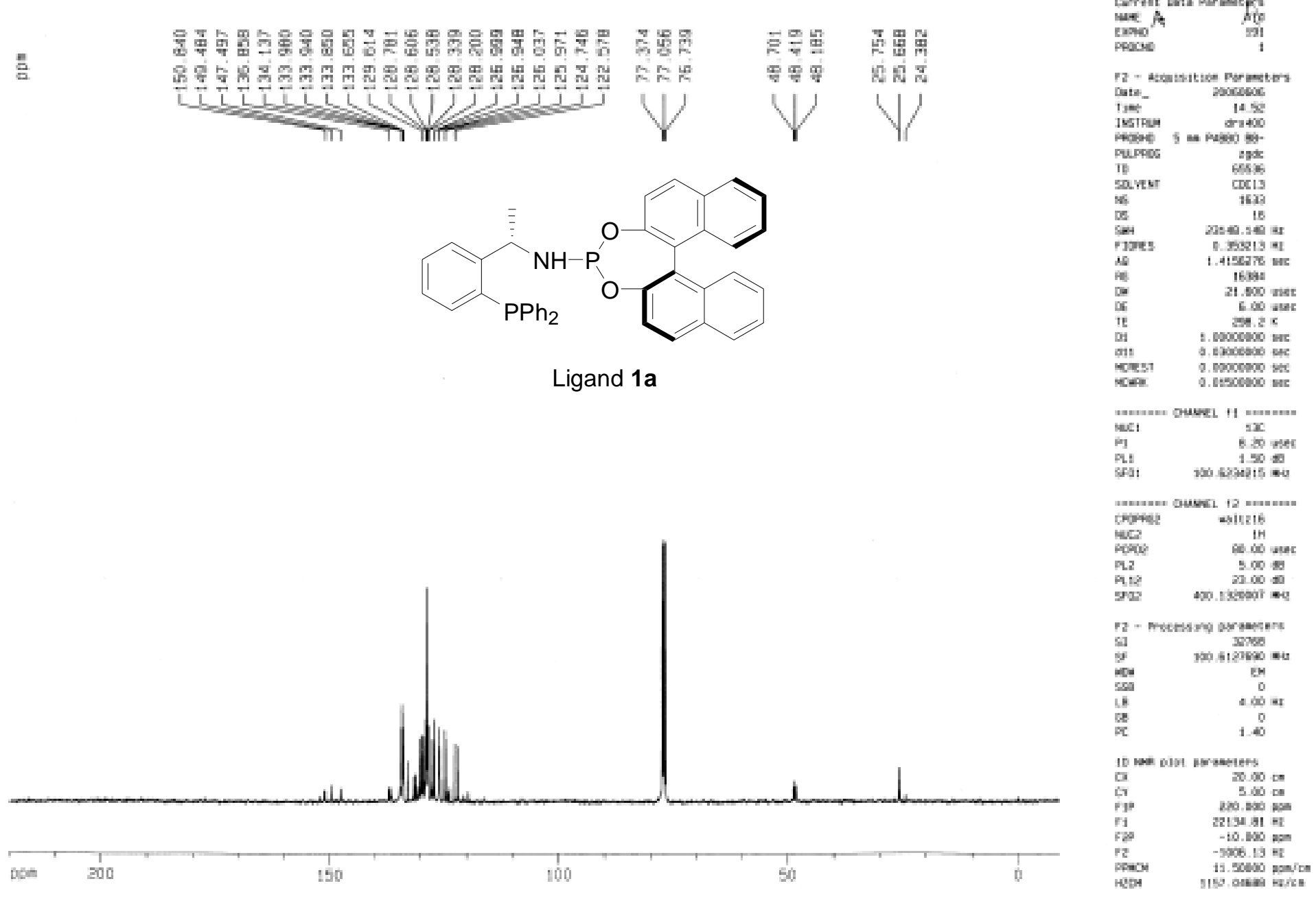


1H RूA KO-1 IN COCL 3 06/05/19

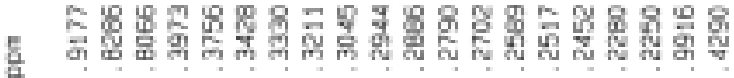

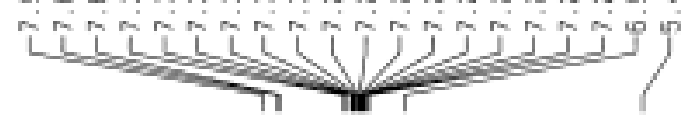

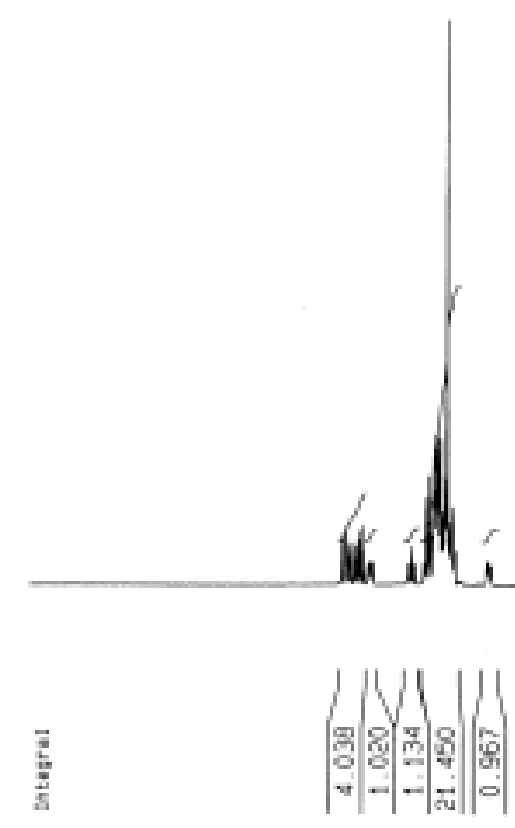

Dpn

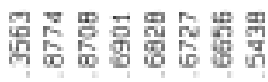
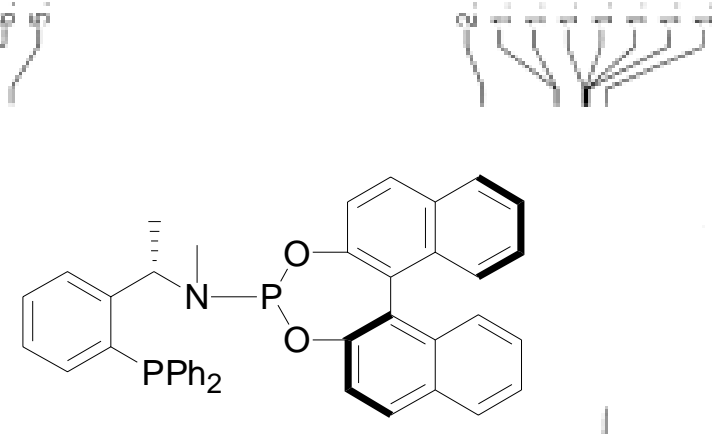

Ligand 1b
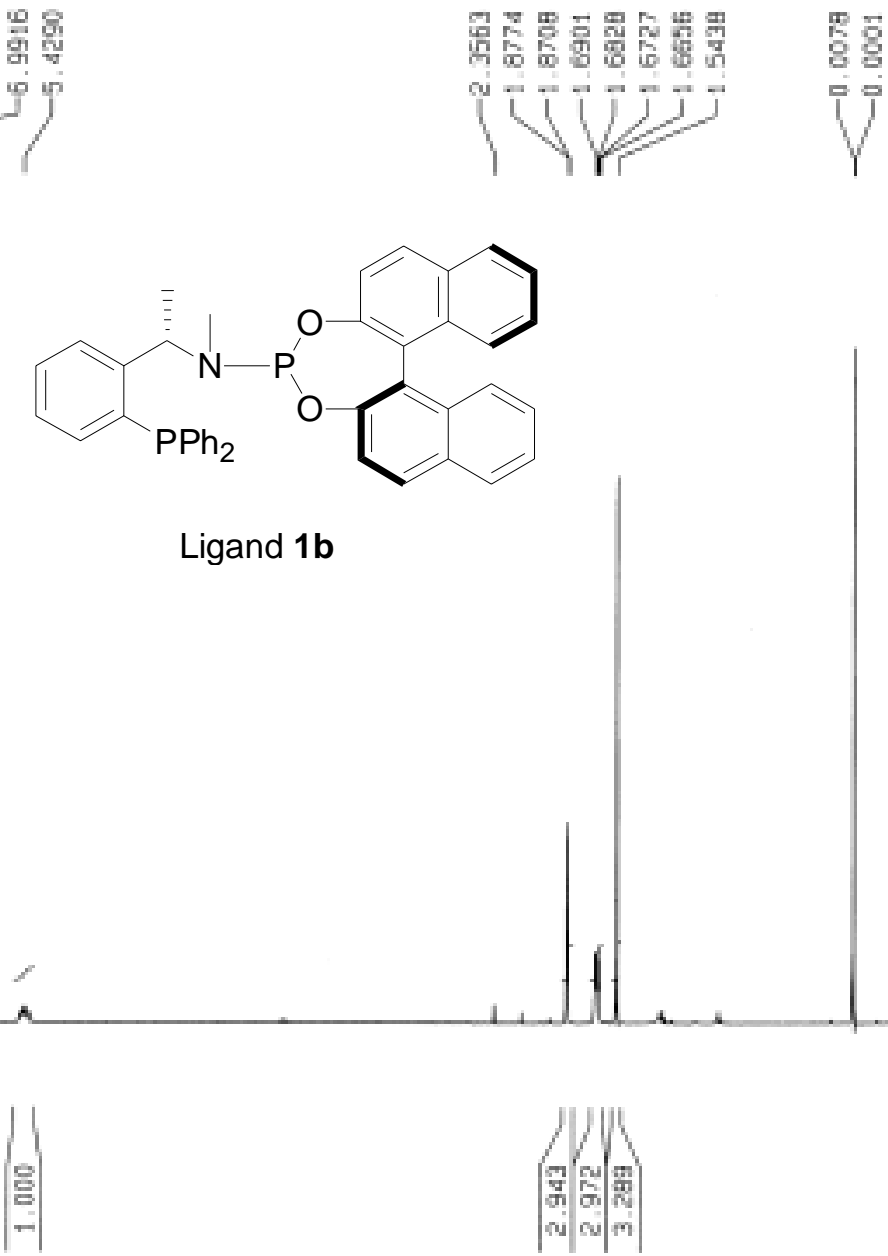

curront 0ot a Poraveters

Mupe $C$

${ }_{100}^{n i d}$

fe - Acqujsition Paraseter:

Date_ 20060519

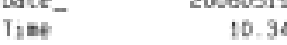

INGTRUM GOA 400

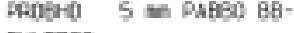

PULF05 29

SOLVENT COC13

16

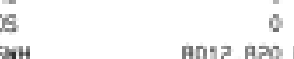

Floces $0.244592 \mathrm{~Hz}$

$40 \quad 2.0447731 \mathrm{set}$

af

ow $\quad 62.400 \mathrm{GanC}$

a $\quad 6.00$ vosec $206.2 \mathrm{~K}$

1. 00000000 set $0.06005050 \mathrm{sec}$ 0.01500050 set CHNeL i1 man...... NuC: $\quad 1$ a. 20 usec Fi1 $5.00 \mathrm{~dB}$ Srot 400.1332521 ตi:

f2 - Processing daranter SI 32768 SF 450.1300177 10i NoN
559 LA $\quad-0.50 \mathrm{HQ}$ $\begin{array}{lr}G B & 0.1 \\ P C & 1.40\end{array}$

10 NMR slot paraneters

$\mathrm{CX} \quad 20.00 \mathrm{ca}$

$\begin{array}{lll}\mathrm{Cr} & 0.00 \mathrm{~cm}\end{array}$

$\begin{array}{lr}\text { F1P } & 10.000 \mathrm{pem} \\ f_{1} & 4001.30 \mathrm{~Hz}\end{array}$

Fap -1.000 pfes

$-450.13 \mathrm{mg}$

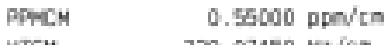

HeCM $220.07150 \mathrm{Ne} / \mathrm{Ca}$ 
31P NMA HND-1 IN CECL 3 06/05/19
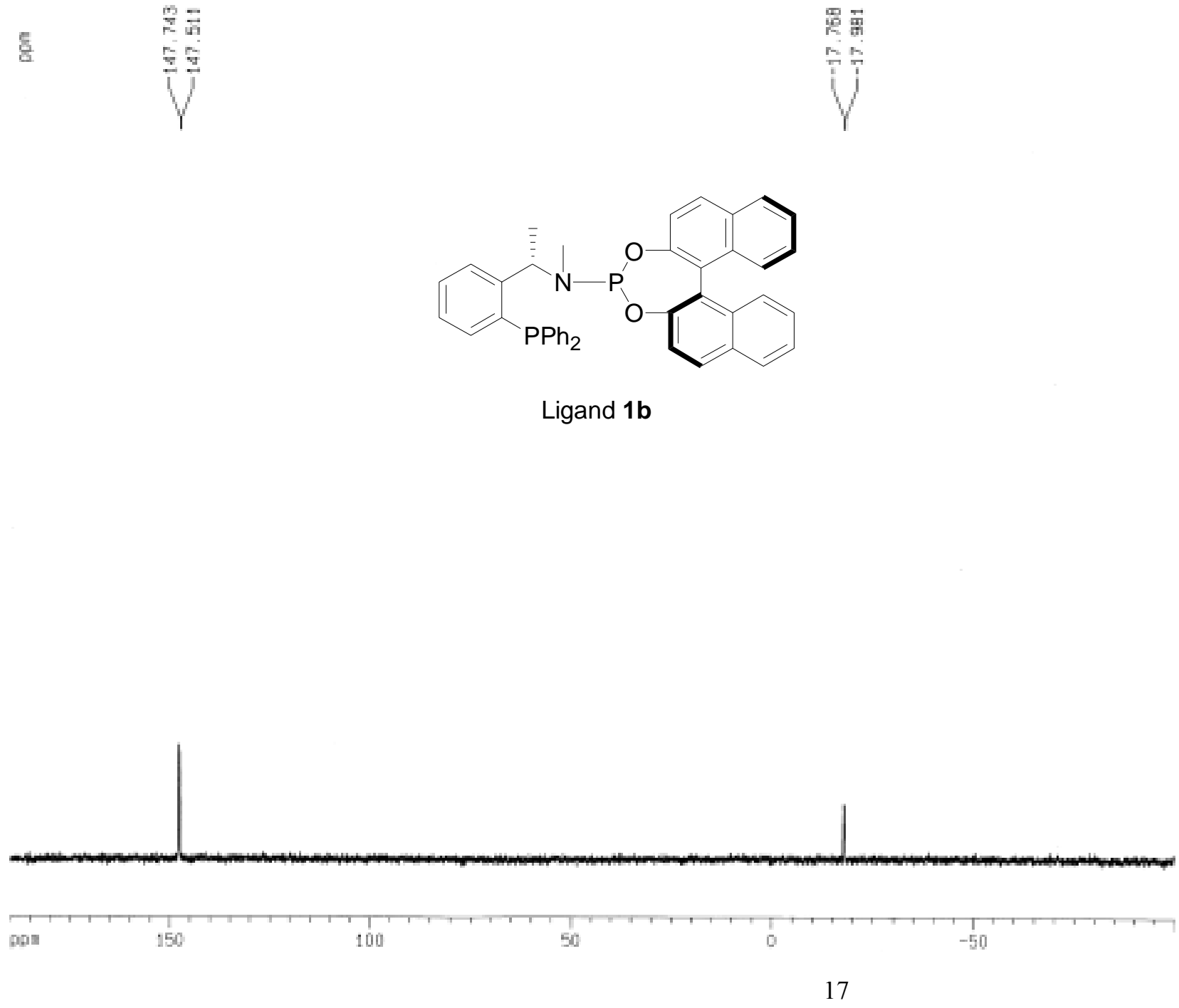

Ourrent Dats Parseters

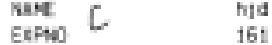
$\operatorname{manc000}$

$\mathrm{Fe}$ - Acoulsution Paremeters

$\begin{array}{ll}\text { date- } & \text { sostosis } \\ \text { The } & 10.41\end{array}$ pacero s a pueso etePUPAP 29 $\begin{array}{ll}\text { ro } & \text { restas } \\ \text { solvent } & \text { Cocis }\end{array}$ 15 Sort 46543.609 He *3 $\quad 0.3975604 \mathrm{sec}$ मag 13004 年 10.300 vit TE ot 2 ecooscon sect

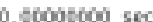
0.01500000 ses

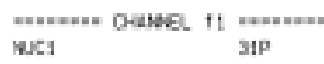
$\begin{array}{ll}\text { wes } & \text { sip } \\ \mathrm{P}_{1} & 8.00 \text { vee: }\end{array}$ A. 161.09250 F2- Pratewaing paraswters.

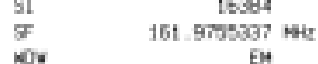
Nor 599

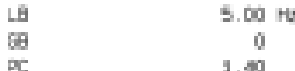
I0 sem plot parameters a $20.00 \mathrm{co}$ 5r $2.00 \mathrm{~cm}$ 190.600 toe

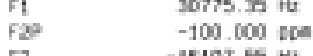

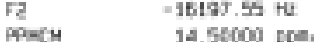
HECM 23eB.62506 He/ch 
13C. NMR H.J-3 IN COCL3 06/06/30

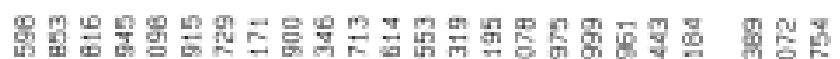

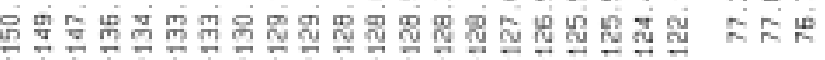

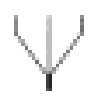

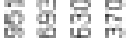

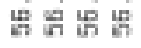

品的赑
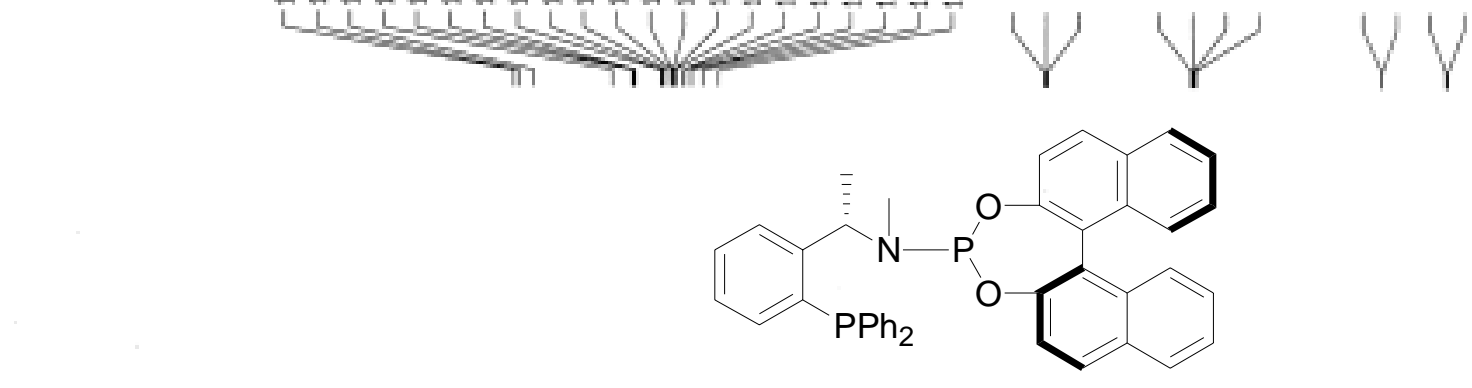

Ligand 1b

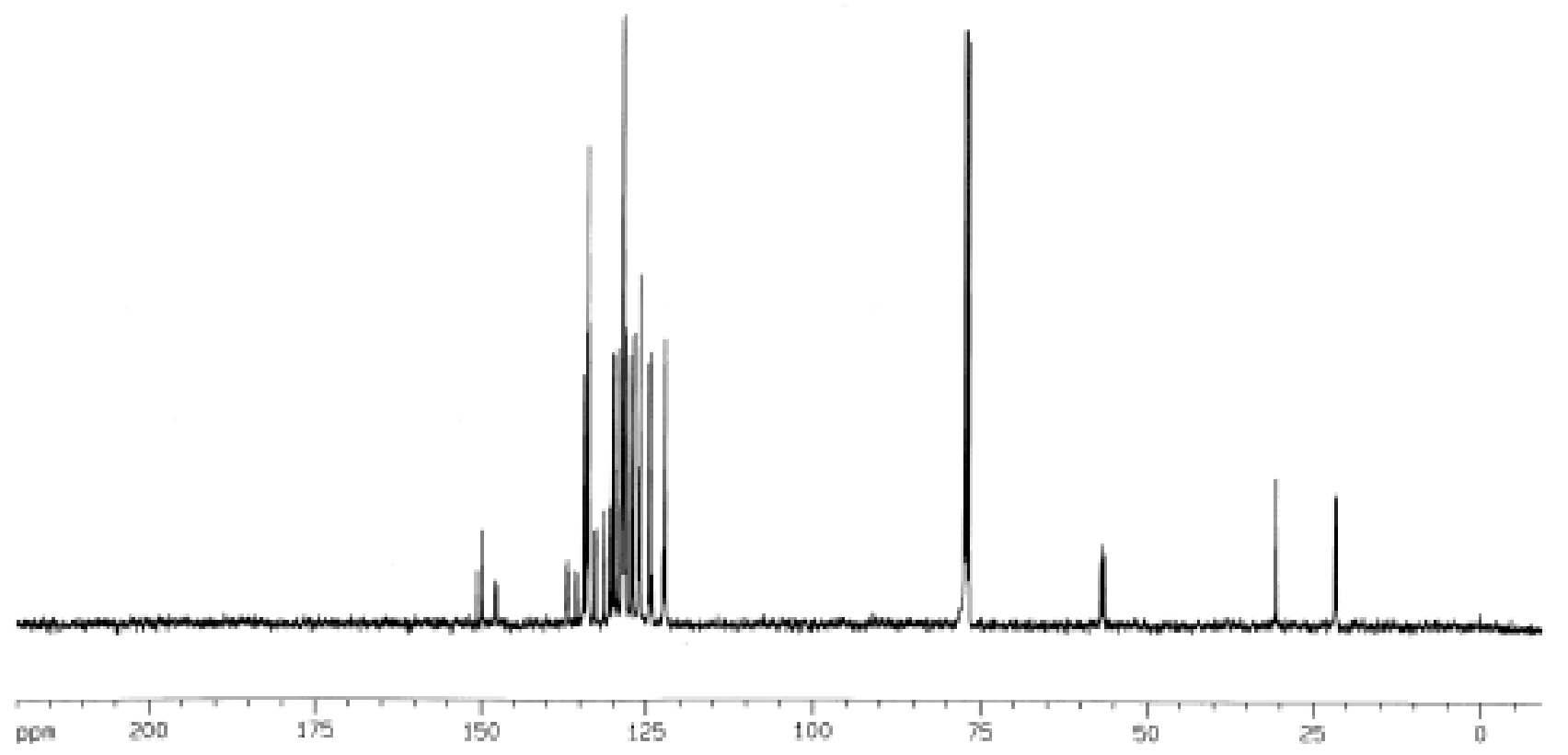

arrent tats Prometers

now $C$ its

Fe - Actusistion Porveterers

Tive-

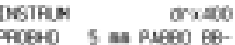

UPFOE

To

SAvem $\quad$ Coc13

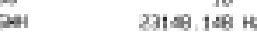

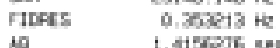

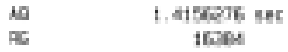

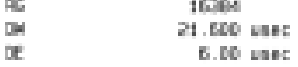

350.25

1. 00000000 set

coest o. oxectecest set

cons 0.0156060 set

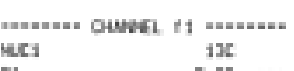

o.

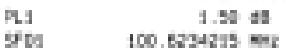

......... owvect is .........

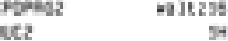

thes $\quad 50.010$ usect

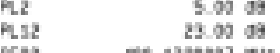

$406.1920 \% 7)$

F2 - Arocessing ongactors

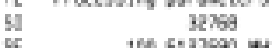

5.

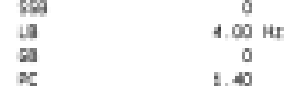

ga net plet zeranetors

$\begin{array}{ll}\mathrm{cr} & 20.00 \mathrm{co} \\ \mathrm{st} & \mathrm{a} .00 \mathrm{co}\end{array}$

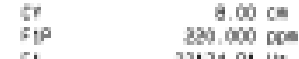

-10.000 pen

t1006. 12 its

FNOA $11.50000 \mathrm{ppe} / \mathrm{t}$. 
IN NMA HJD-3 IN COCL3 06/06/06

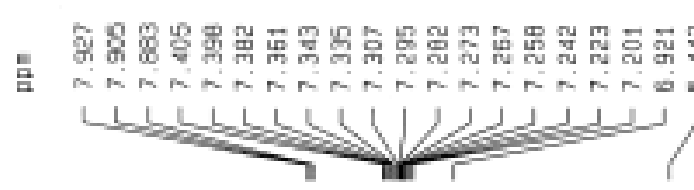

要要哭要

4

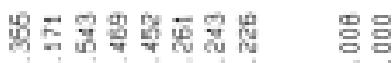
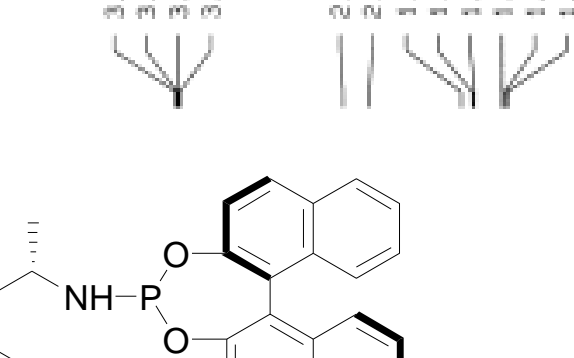

$\mathrm{PPh}_{2}$

Ligand 1c

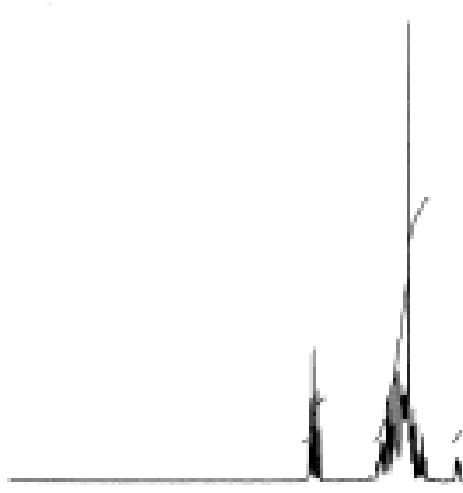

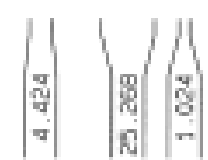

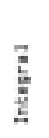

$\mid$

ppo
Curfent Dete Paraseters

wee hid

Exe- 0

F2 - Acouisitian Parcoeter:

Date

Tine 20060006

1NSTRUM Or:

PULPFOS

29
35258

SOLVEN

18

tos

FLDFES $\quad 0.240532 \mathrm{HE}$

40 $2.0447731 \mathrm{sec}$

P6 256

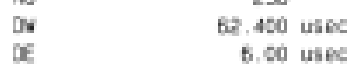

6.00 usec
$297.2 \mathrm{~K}$

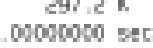

$\begin{array}{ll}\text { MCEEST } & 0.000000000 \mathrm{sec} \\ \text { MCNFK } & 0.01500000 \mathrm{sec}\end{array}$

awel is ..........

WuCS $1 \mathrm{H}$

P.1 5.1200

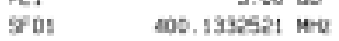

F2 - Processung dor uepters

51 12708

F $\quad 400.1300182 \mathrm{~B}$ M-

NOW GM

SSB $-0.50 \mathrm{H}$

al 20.1

10 has plot soraneters

Cr $20.60 \mathrm{~cm}$

t5. 6. $\quad 600 \mathrm{cos}$

ri 10.000 pas

F2P

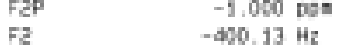

$\begin{array}{ll}\text { PFMCM } & 0.50000 \mathrm{DON} / \mathrm{cm} \\ \mathrm{HZOH} & 220.07150 \mathrm{~Hz} / \mathrm{Cm}\end{array}$ 
31P NAR H.O-3 IN CDCL3 O5/06/05

言

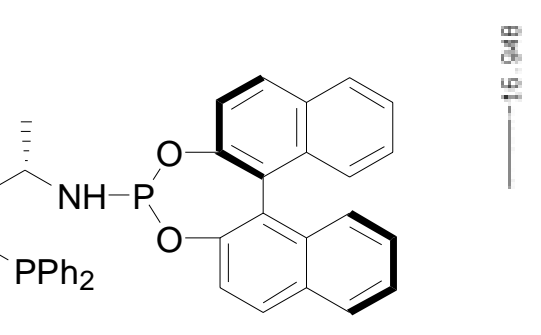

Ligand 1c
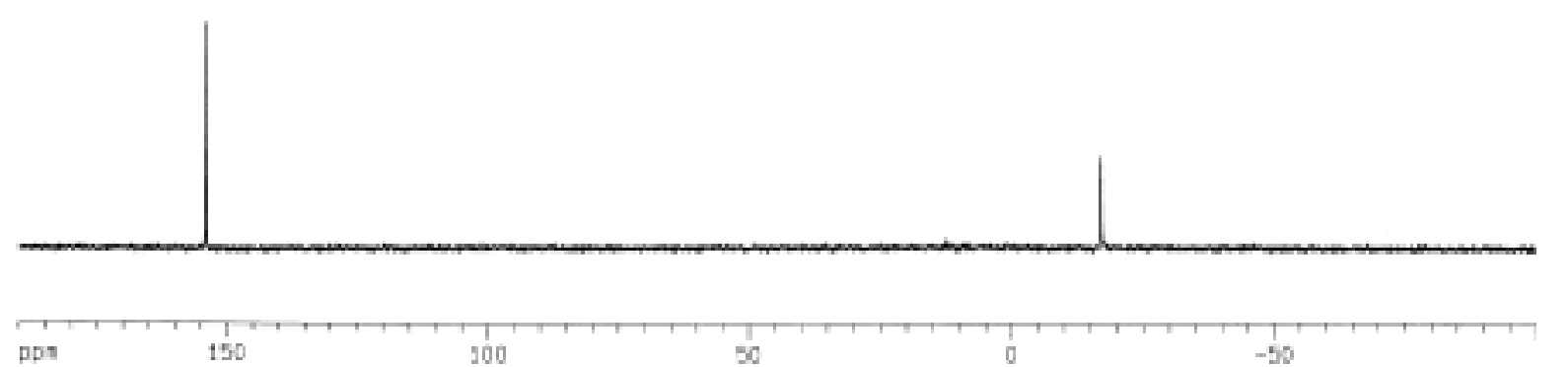

150

$\frac{11}{900}$

${ }_{s 0}$

$\int_{-50}^{1}$

Turreme dota Porueters

Expen

F2 - Acquisition pyoneters

bete_ 20050506

1100

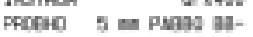

PUPAOS

To
savert exis
exis

16

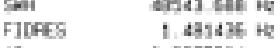

$20 \quad 0.3395942$ set

to n. 390 usec

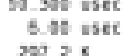

2.00050000

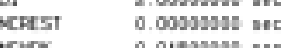

KCFF. 0.01505000

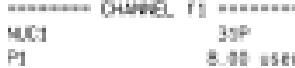

in $0.00 \mathrm{ats}$

F2 - Processing parsosters

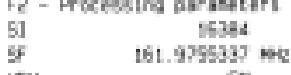



58
68

10 nee ploc asmometers

a $20.00 \mathrm{~cm}$

rip 100000

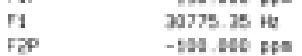

Fe

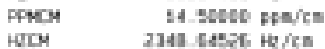




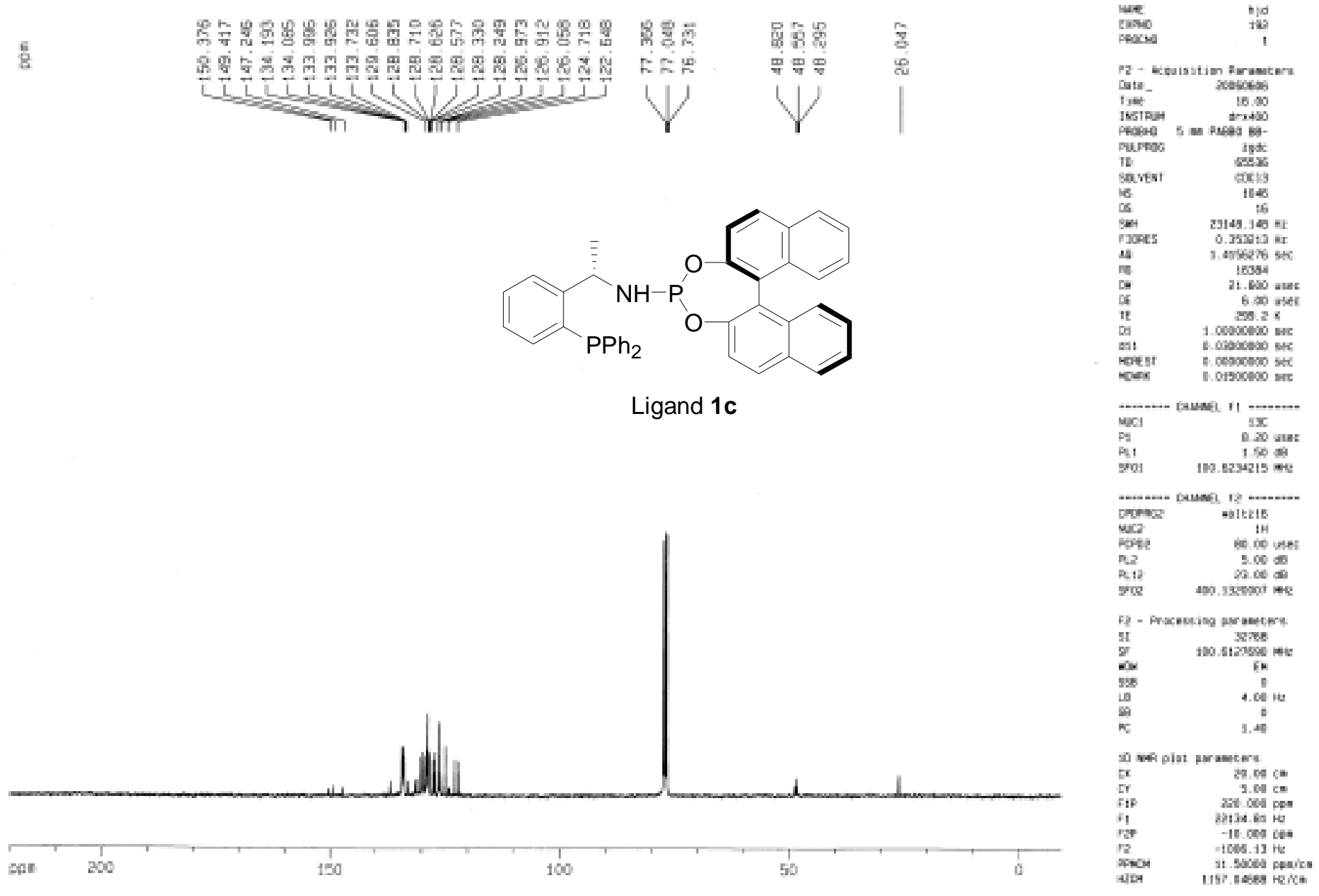


1H NMA H. H-2 IN CDO-3 06/05/19

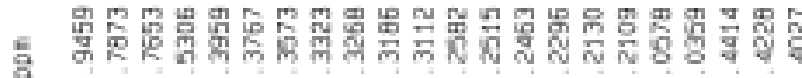
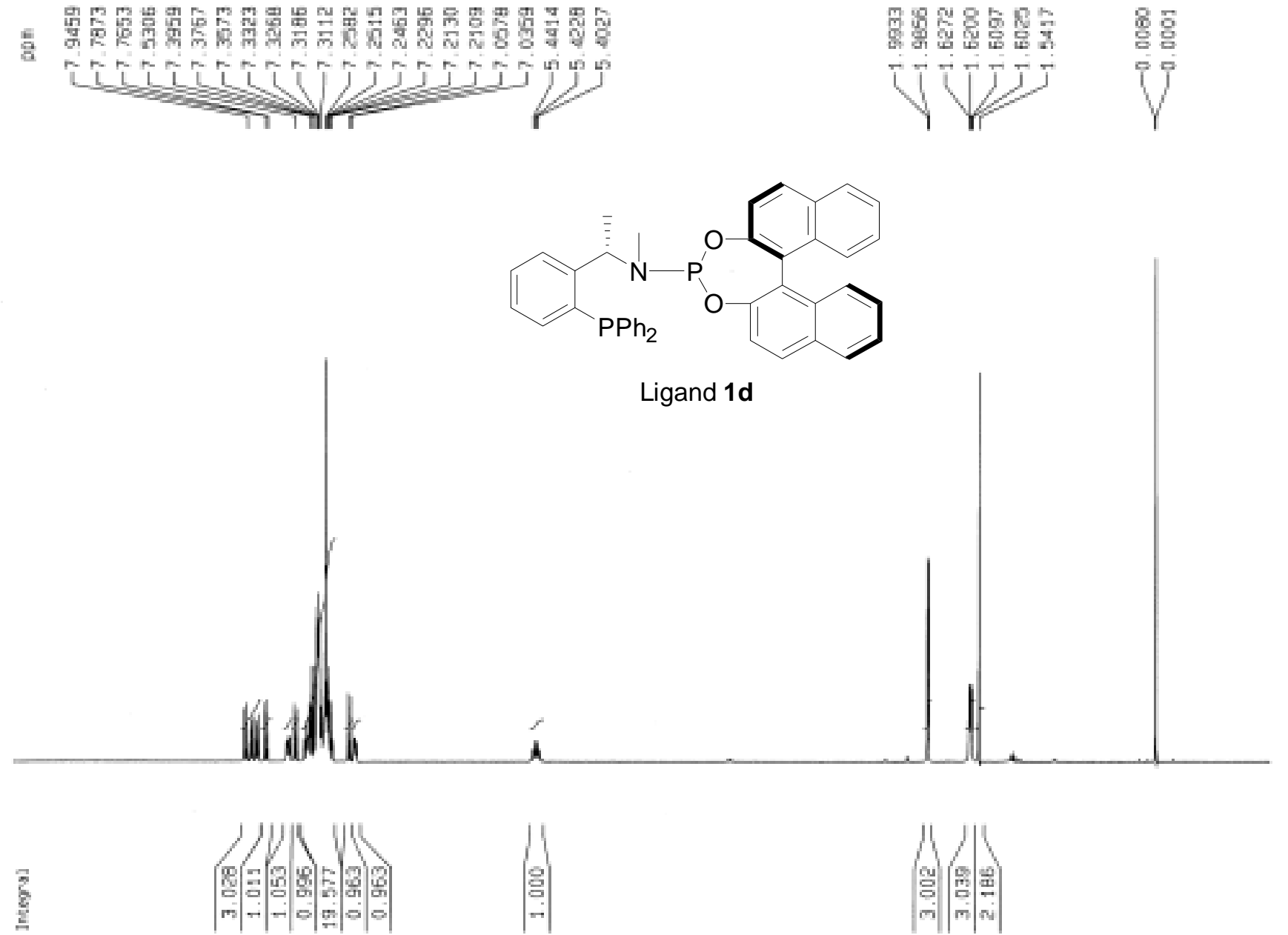

|

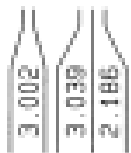

Curfent Data Paraneters NAE D his PAOCN

F2 - Acouisjtion Paraneters

Date_ 20060sig

ine $\quad 10.47$

INSTRMA or $x 200$

CACENO $5 \mathrm{~mm}$ PABSO $20-$

PUFAOG

gavent Coc19

SW-1 $8012.800 \mathrm{~Hz}$

IDFES $\quad 0.244532 \mathrm{~Hz}$

$18 \quad 2.0447731 \mathrm{se}$

18018

DW 62.000 usec

CE 6.00 USAC

TE $295.2 \mathrm{~K}$

O1 $\quad 1.00000000 \mathrm{sec}$

C.FF. $\quad 0.00000000 \mathrm{sec}$

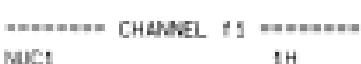

$\begin{array}{ll}\text { MuCt } & \text { IH } \\ \text { P1 } & \text { 8.20 usec }\end{array}$

(1) $5.00 \mathrm{de}$

SFO1 400.1332521 $\mathrm{mHz}$

F2 - Processing paraneters

Si 1 32)

SF $\quad 400.1950179 \mathrm{kHz}$

NDN GM

$-0.50 \mathrm{HS}$

$\begin{array}{rr}G \theta & 0.1 \\ P C & 1.40\end{array}$

10 MMe duot Daranters

$\mathrm{Cx} 20.00 \mathrm{ct}$

$\mathrm{Cr} \quad \mathrm{B} .00 \mathrm{~cm}$

Fip $\quad 10,000$ ote

$\mathrm{F1} \quad 4001.30 \mathrm{kz}$

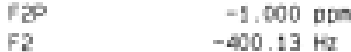

PPNCM $\quad 0.55600 \mathrm{ppon} / \mathrm{cos}$

HOCM $220.07150 \mathrm{~Hz} / \mathrm{cm}$

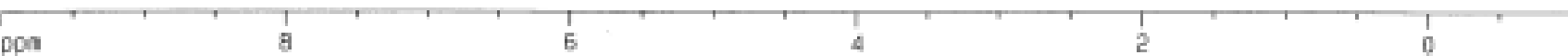


31P NAP HO-2 IN COCL3 06/66/19

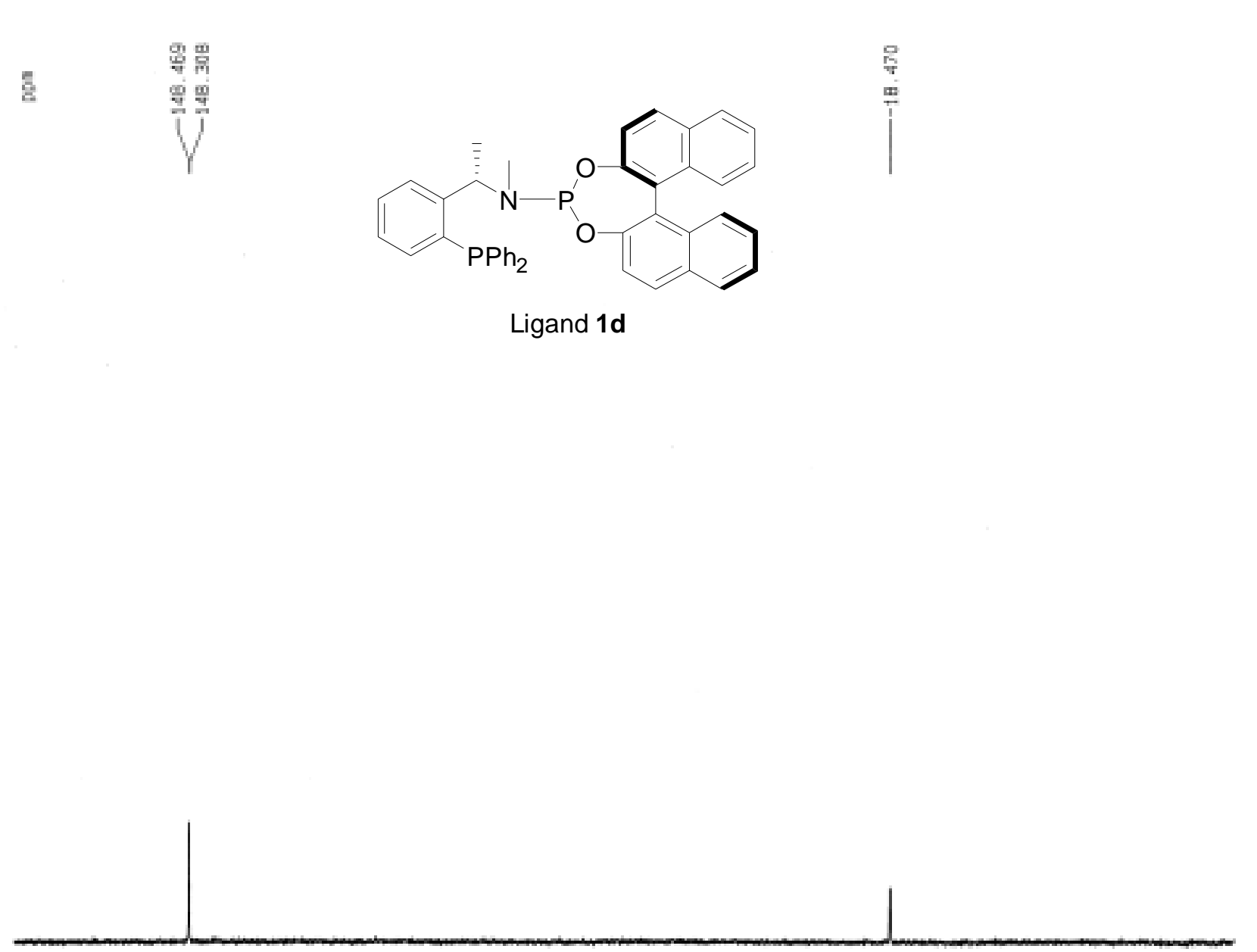

Curfent, fota Paraseters

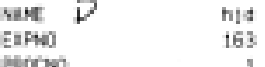

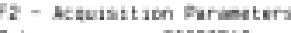

ate- 20060619

(10) 10.53

screo $5 \mathrm{~m}$ pMaso $\mathrm{ag}$ -

Pupros $5 \mathrm{~m}$ PMaso its

Solvent $\quad \operatorname{coc} 13$

$16 \quad 109$

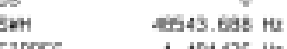

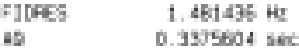

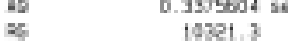

8 . 50.900 usec

II $296.2 \mathrm{~K}$

CPEST

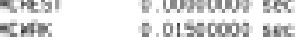

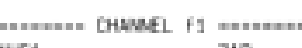

nect 310 ved

0.00
0.020

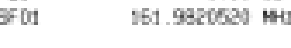

62 - Avocessing pyraeters

95.96504

Co.

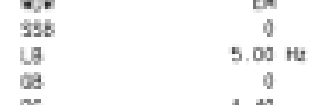

10. Mef gles parameters

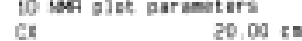

$\begin{array}{ll}\text { Ct } & 20.00 \mathrm{~cm} \\ \mathrm{Ct} & 2.00 \mathrm{co}\end{array}$

$\begin{array}{ll}\text { Fip } & 190.000 \mathrm{kPm} \\ \mathrm{Fi} & 30775.35 \mathrm{ke}\end{array}$

$\mathrm{Fb} \quad-100.000 \mathrm{mpm}$

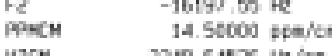

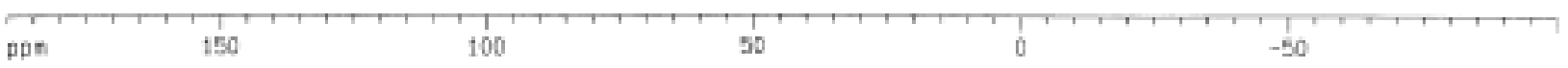

$-50$

2048. $54506 \mathrm{He} / \mathrm{cm}$ 
13C NA HJD-4 IN CDCL3 05/05/30

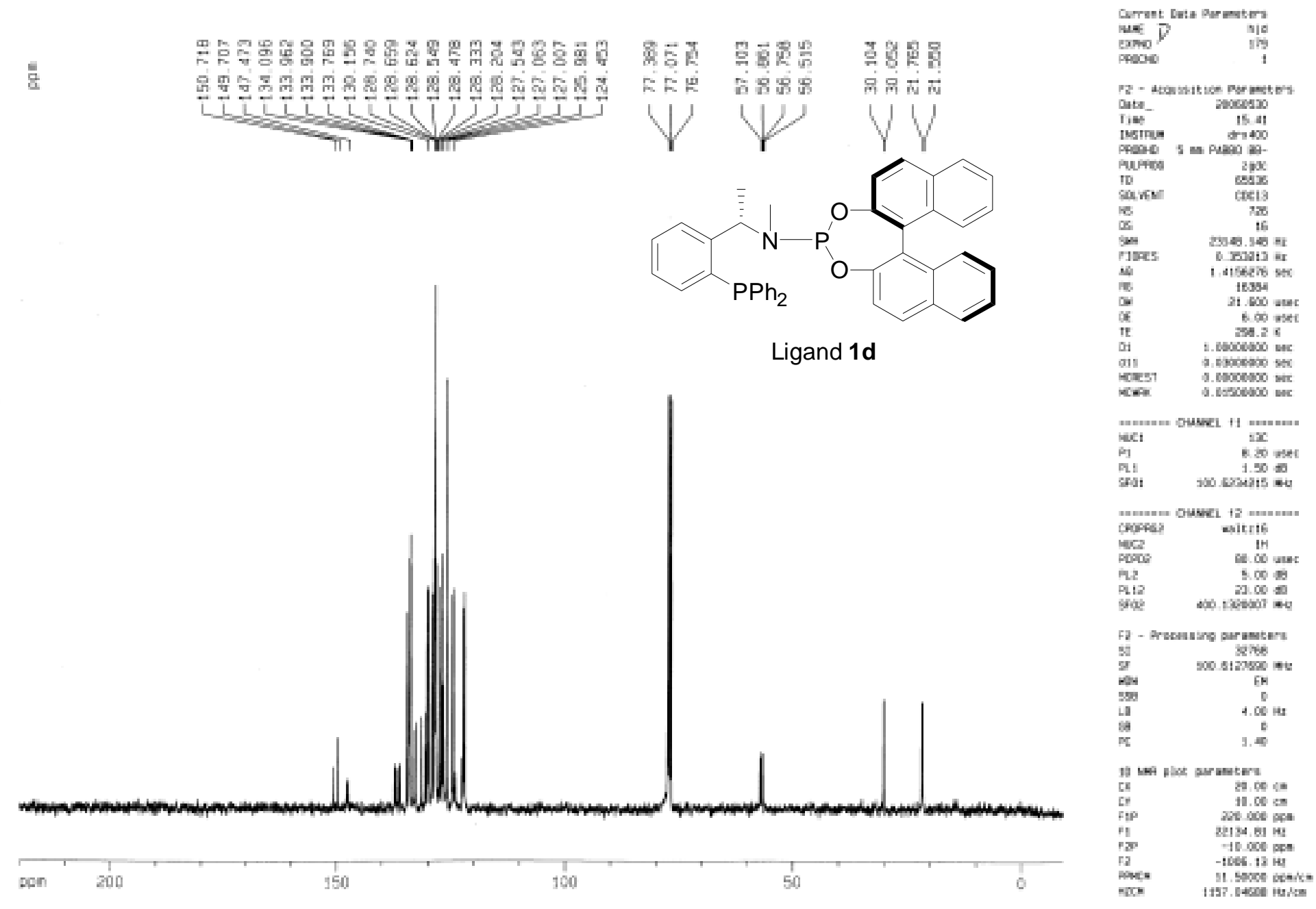



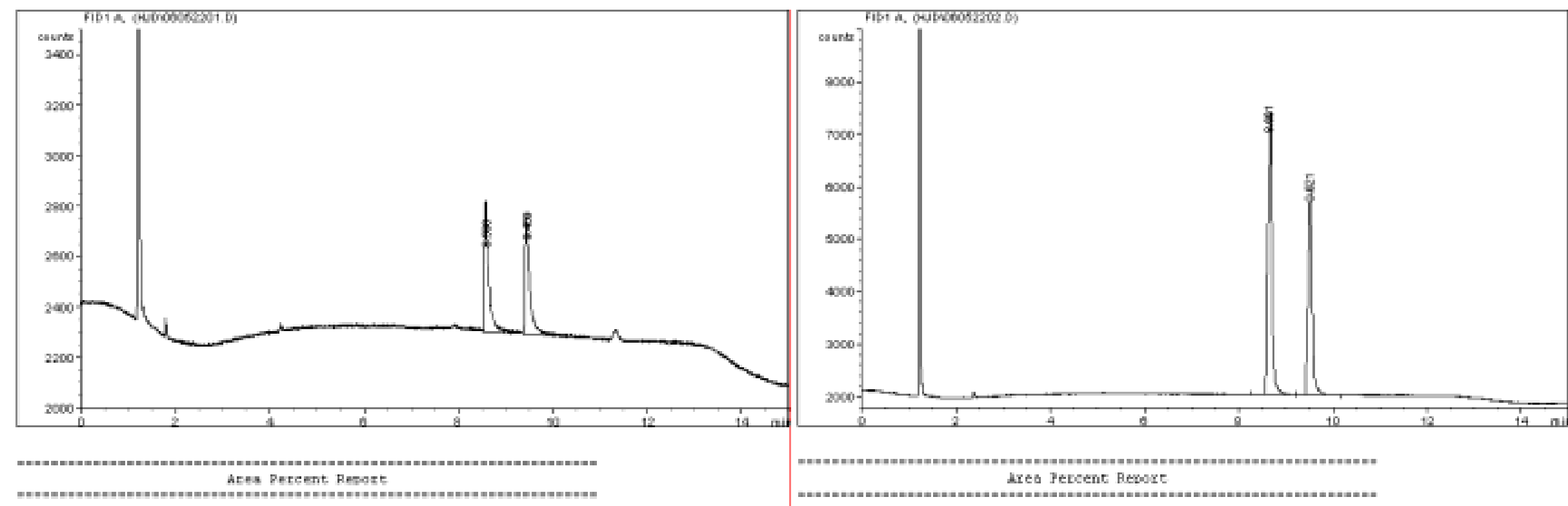

\section{$\begin{array}{lll}\text { Sorted By } & \vdots & 310091 \\ \text { Maltiplier } & \vdots & 1.0000 \\ \text { Diluecion } & 1.0000\end{array}$}

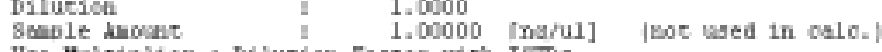

Use Haltiplier \& Dilution Foctor with I I Tha

\section{3agnal 1: rtp1 A.}

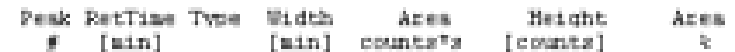

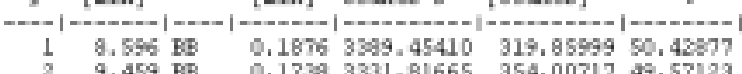

Totals 1 6721.27075 673,86716

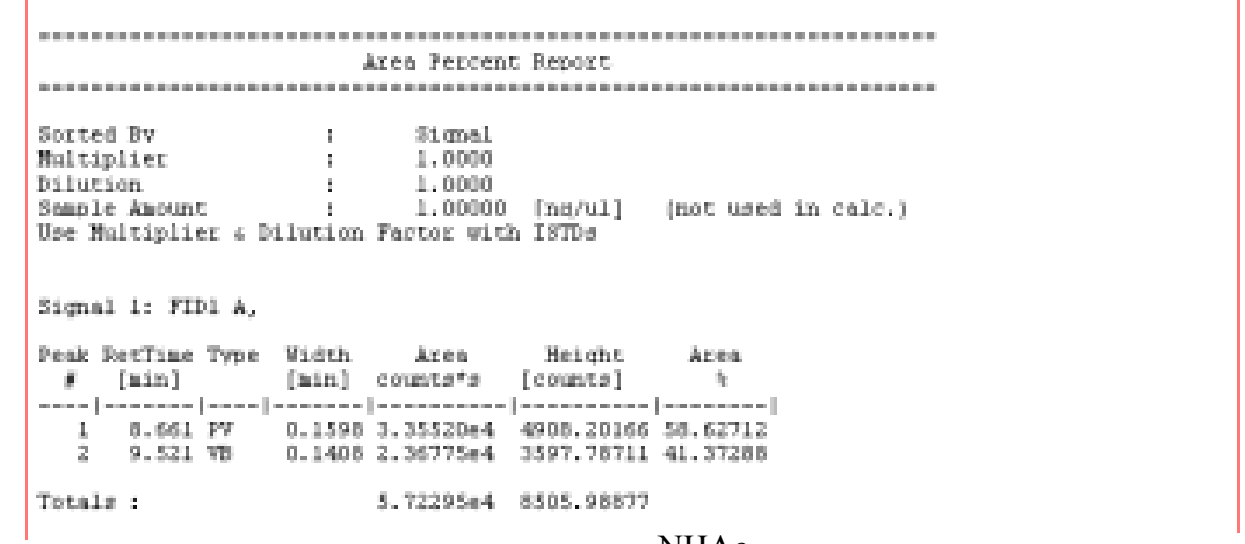

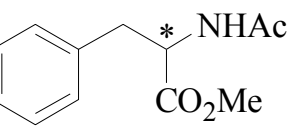

(racemic product)

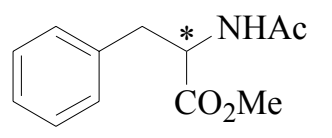

(hydrogenation product with PEAphos 1a)

Entry 1, Table 1 

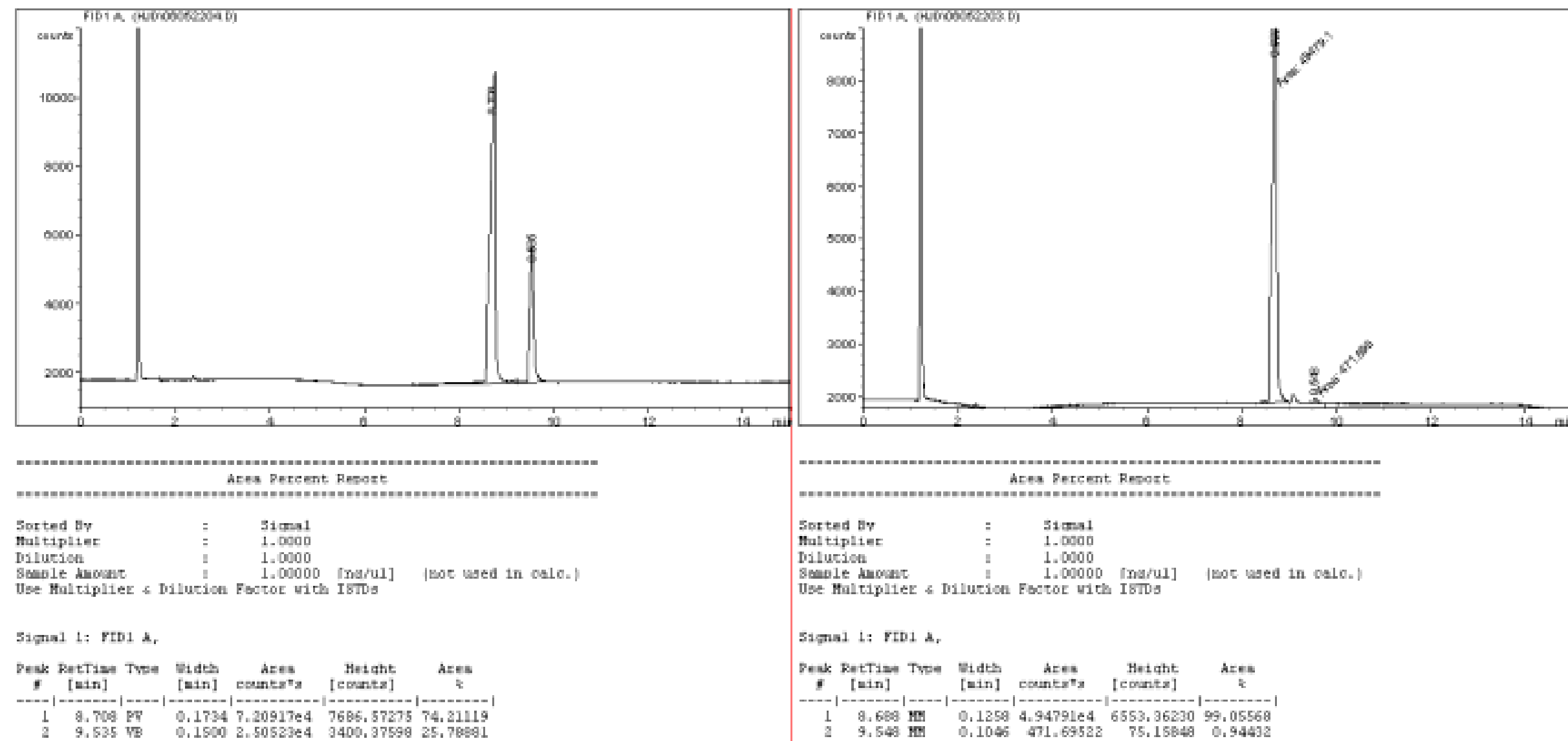

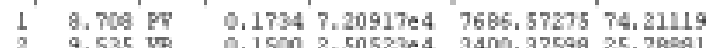

Totals ।

$$
\text { 9.71439e4 1.10969e4 } \mathrm{CO}_{2} \mathrm{Me}
$$

(hydrogenation product with PEAphos 1b)

Entry 2, Table 1
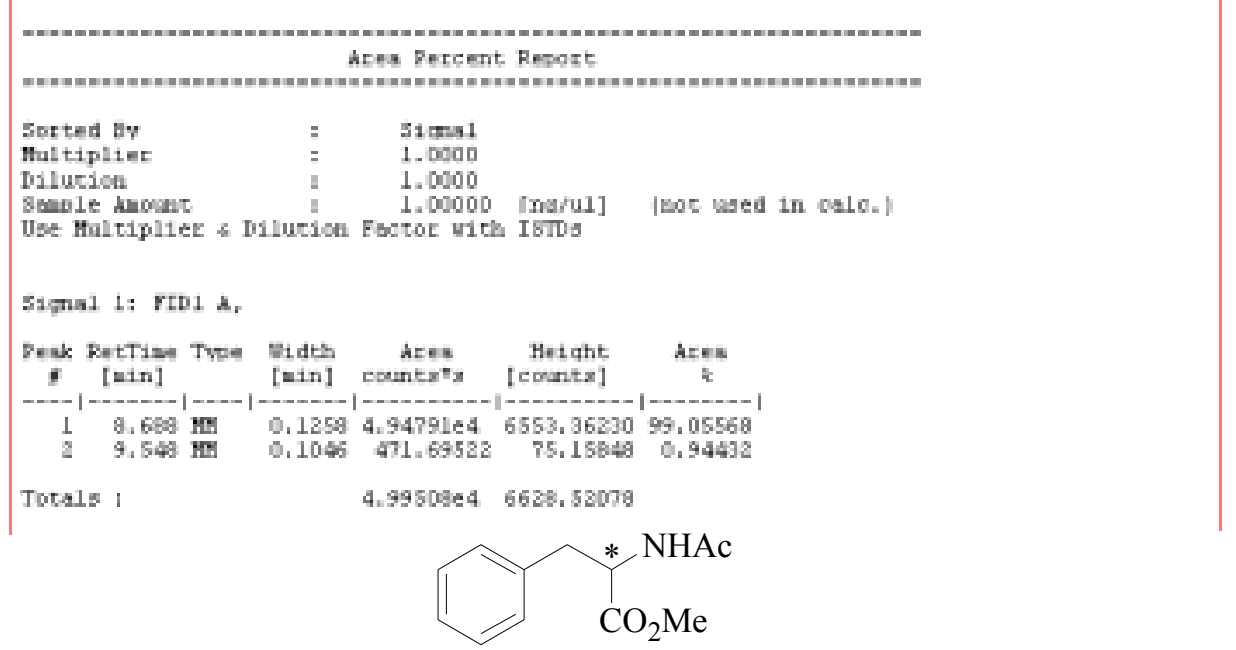

(hydrogenation product with PEAphos 1c)

Entry 3, Table 1 

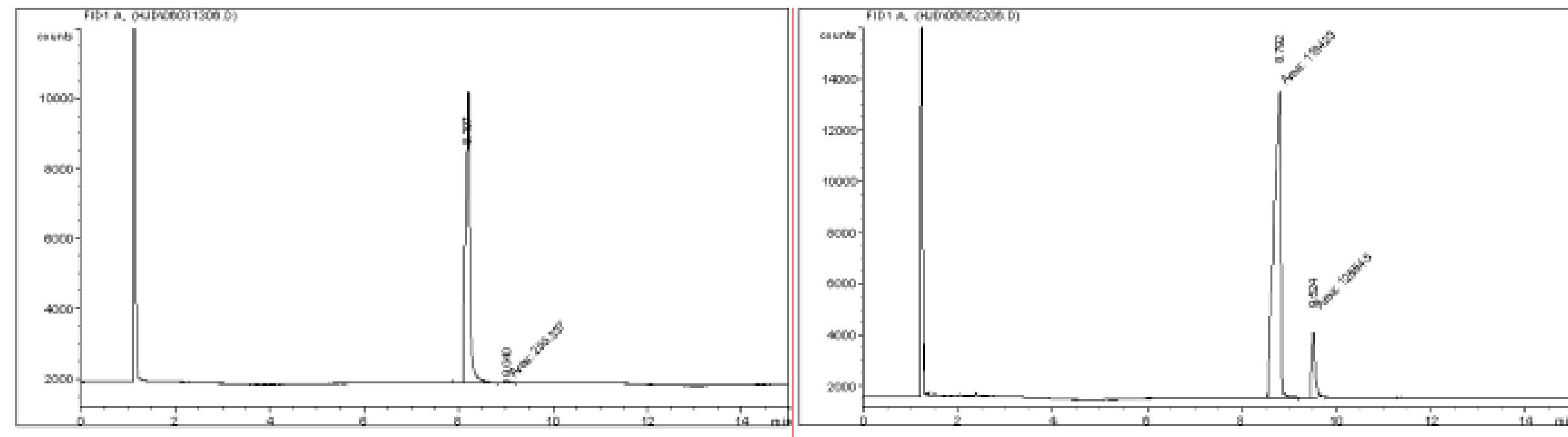

dreo Percent Regorr

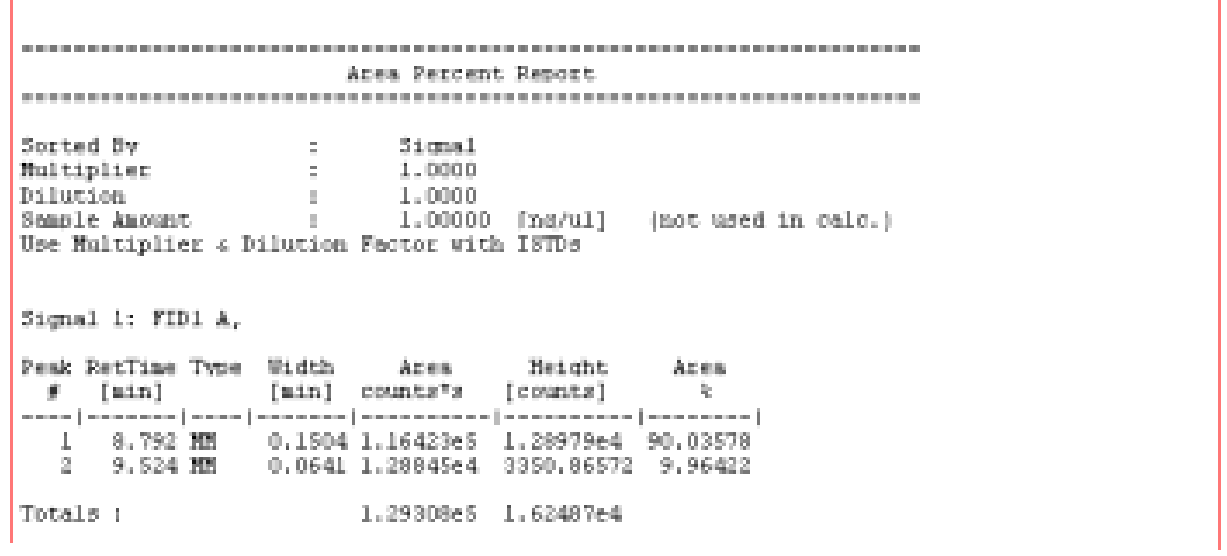

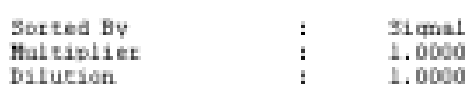

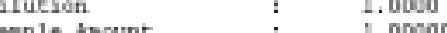

Somple daount in l,00000 ina/u1] (not uses in cale,

Signal in FIDI A,

Peak Rectime Twe Hidth
[min]

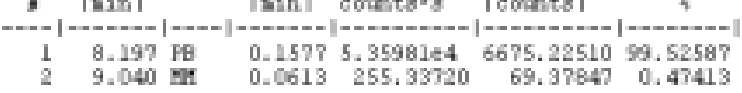

Totals ।

\begin{abstract}
5. 39535e4 6744.60357
\end{abstract}

\section{* NHAc \\ $\mathrm{CO}_{2} \mathrm{Me}$}

(hydrogenation product with PEAphos 1d)

Entry 4, Table 1

\section{* NHAc \\ $\mathrm{CO}_{2} \mathrm{Me}$}

(hydrogenation in PhMe)

Entry 5, Table 1 

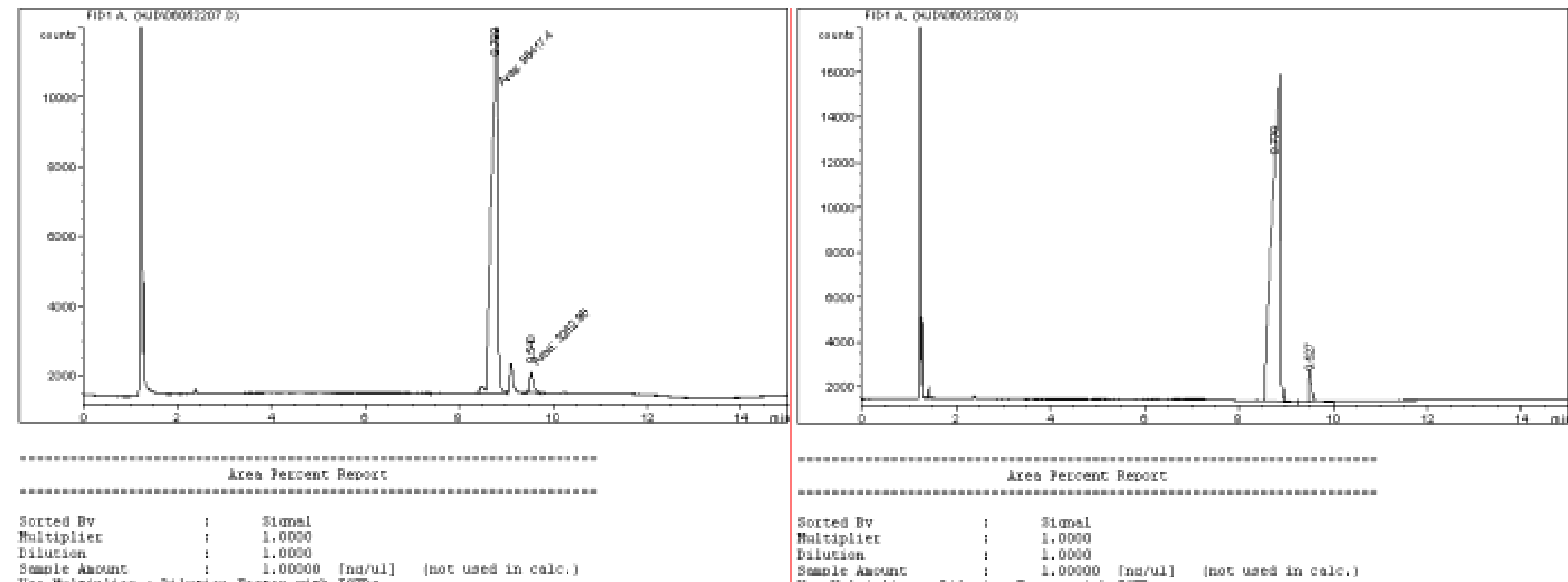

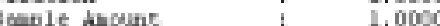

Use Haltipliet o Dilution Factor with ISTd

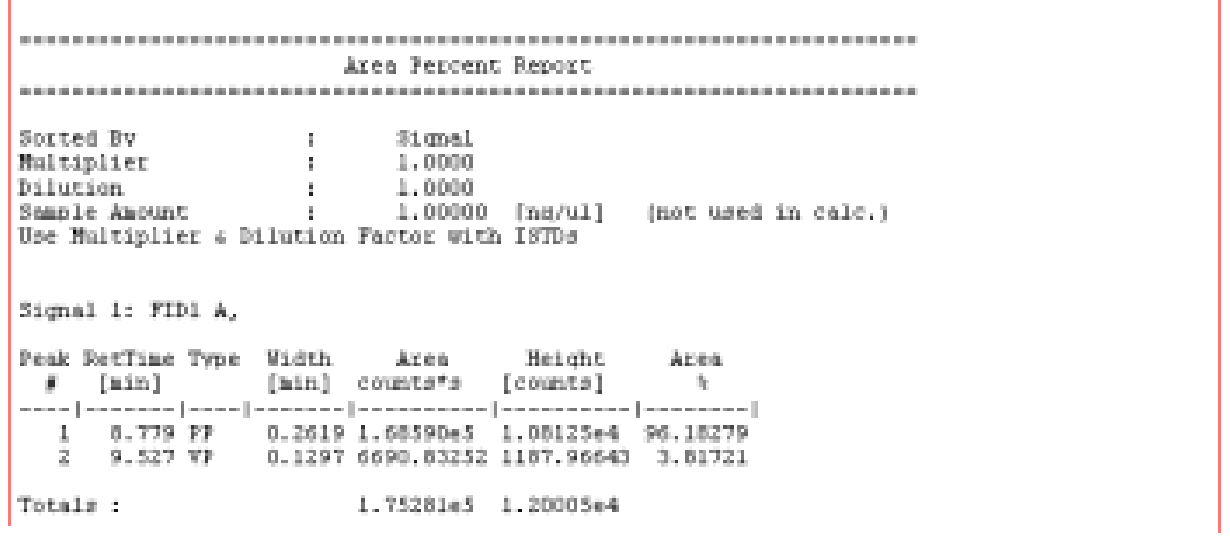

Signal 1: rrDi $\mathrm{A}$

Peak hecTime Trpe Wiath
$[$ [uin]

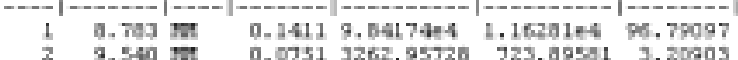

Totalz:

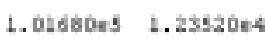

\section{* NHAc \\ $\mathrm{CO}_{2} \mathrm{Me}$}

(hydrogenation in $\mathrm{MeOH}$ )

Entry 6, Table 1

\section{* NHAc \\ $\mathrm{CO}_{2} \mathrm{Me}$}

(hydrogenation in EtOAc)

Entry 7, Table 1 

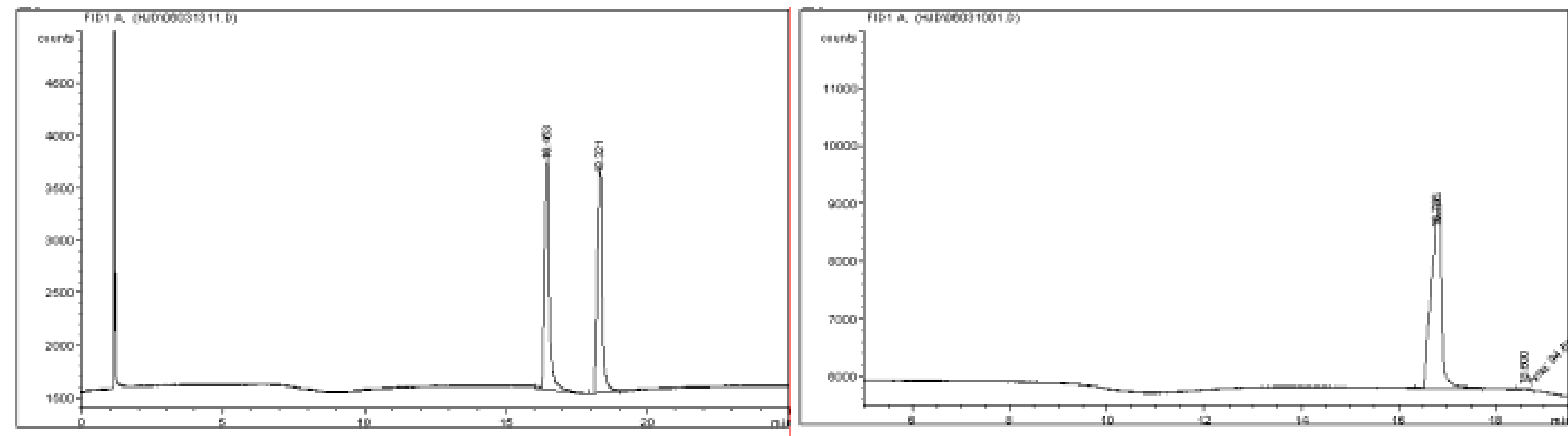

Ares Percent Report

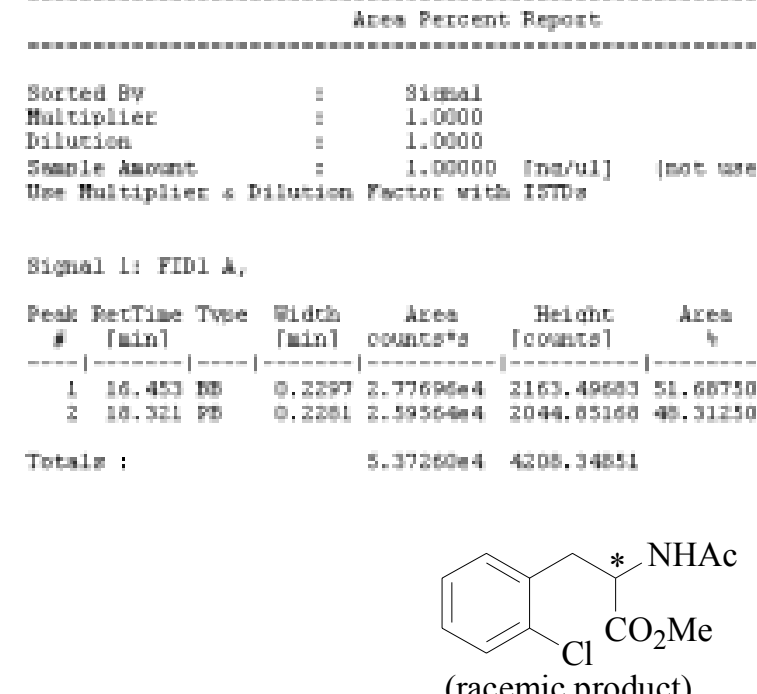

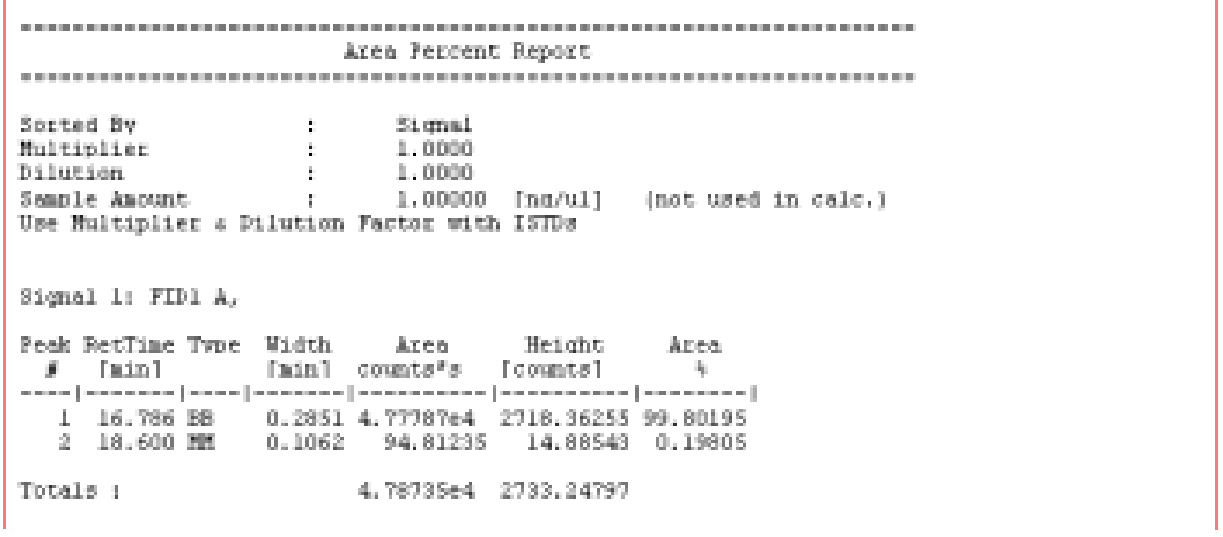

(racemic product)

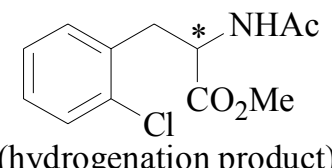

Entry 8, Table 1 

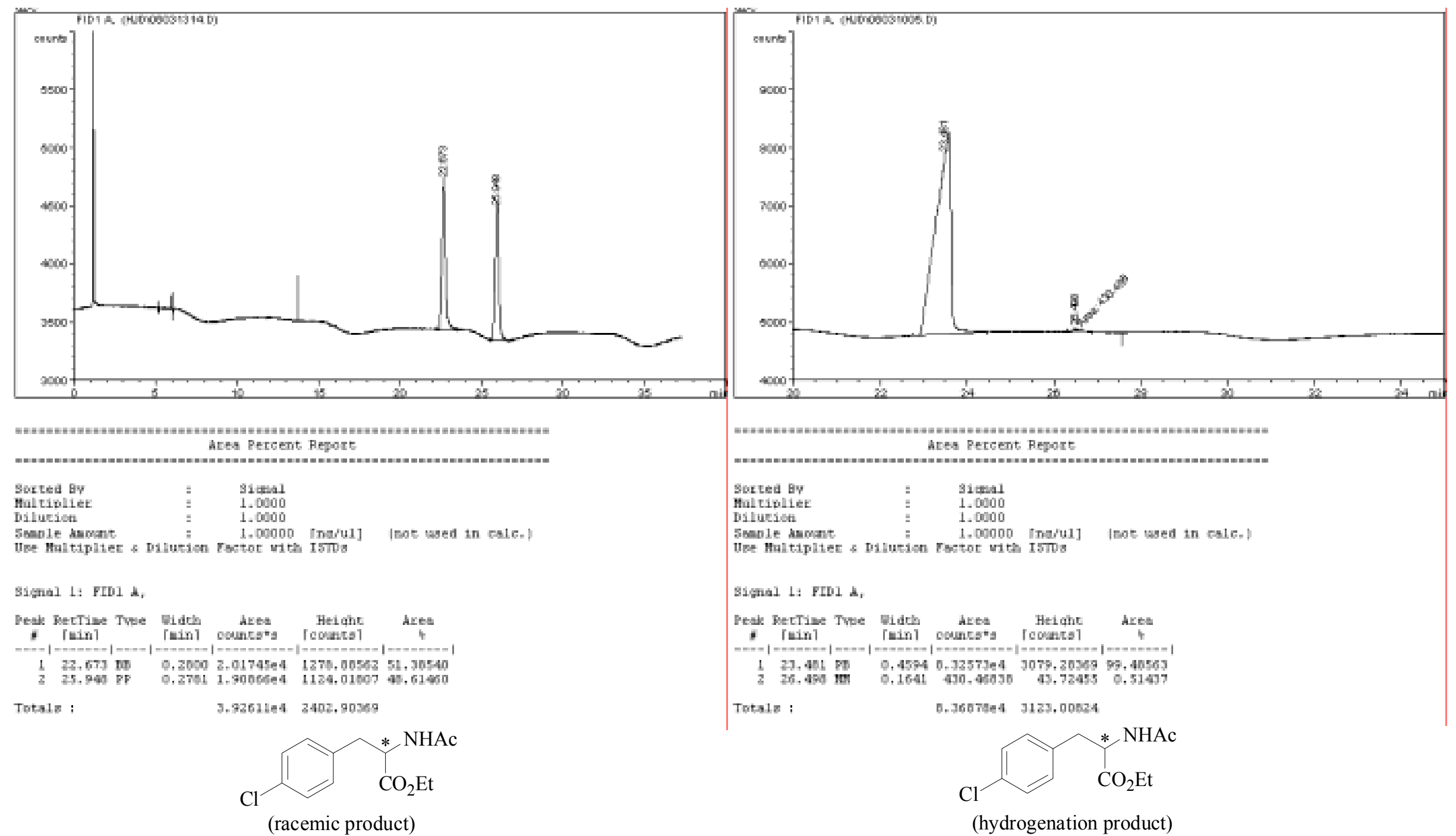

(racemic product)

(hydrogenation product)

Entry 9, Table 1 

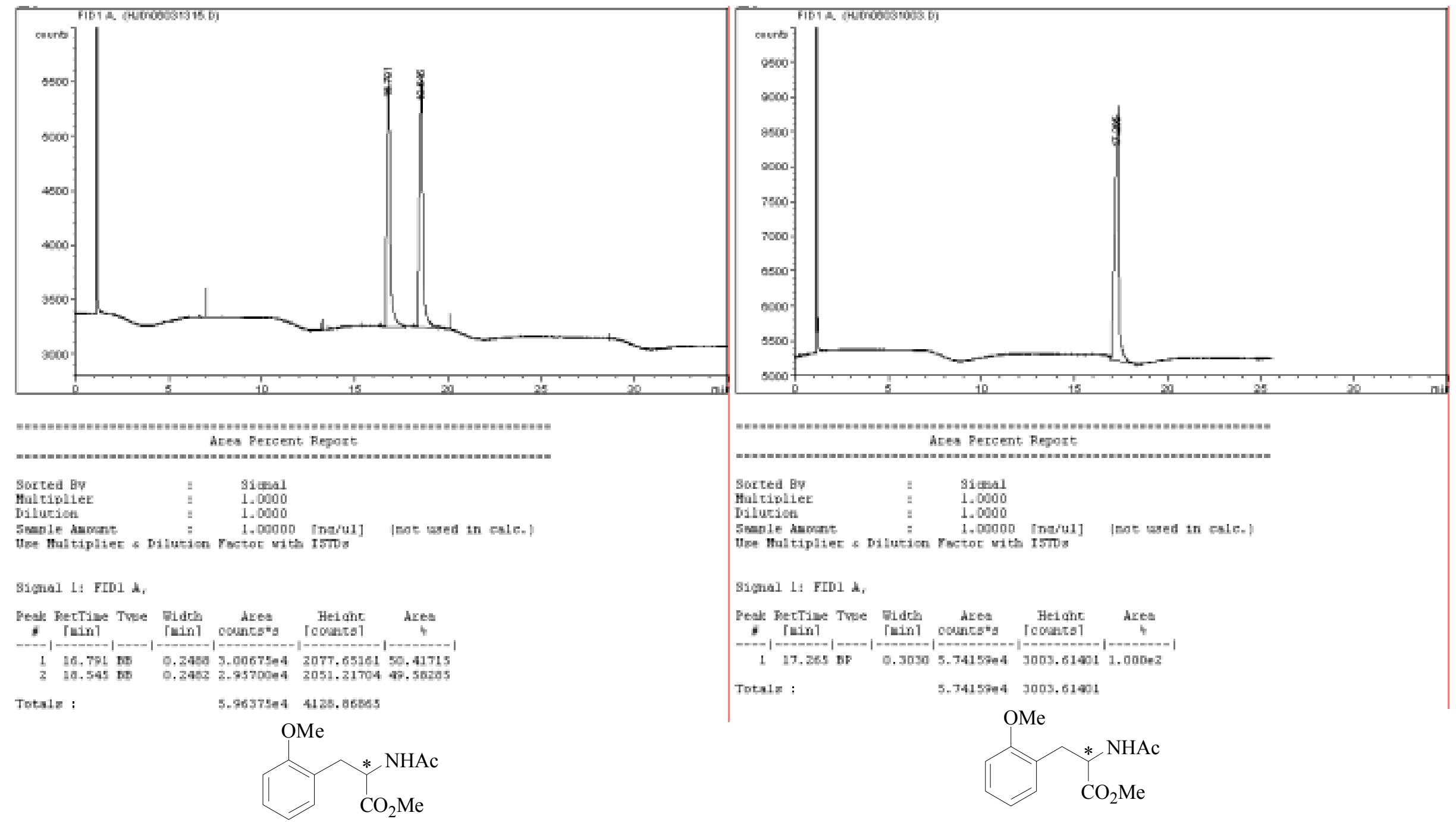

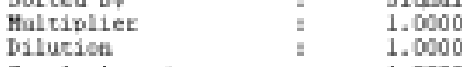

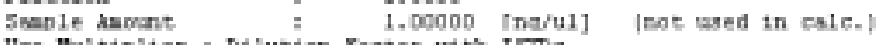

Signal 1: FIDI A,

Peal: hectiale Twe Fidis Area Height drea

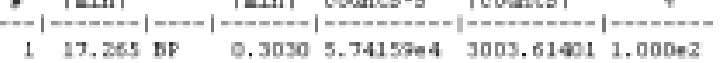

Tota18 : $\quad 5.7415904$ J003,61401

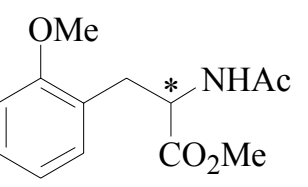

(racemic product)

(hydrogenation product) 

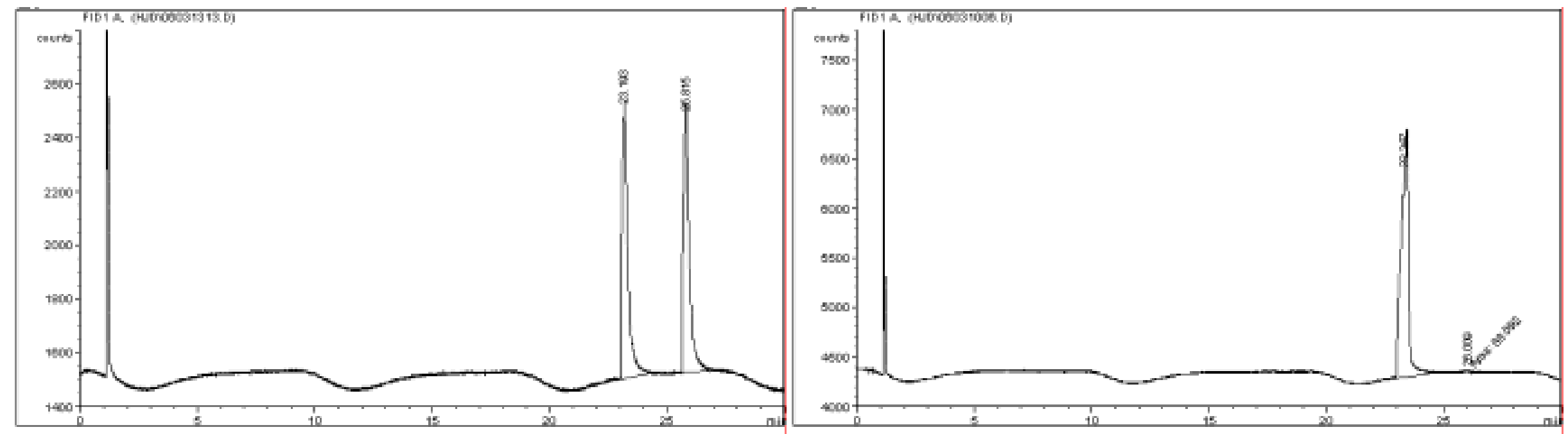

Ares Percent Report

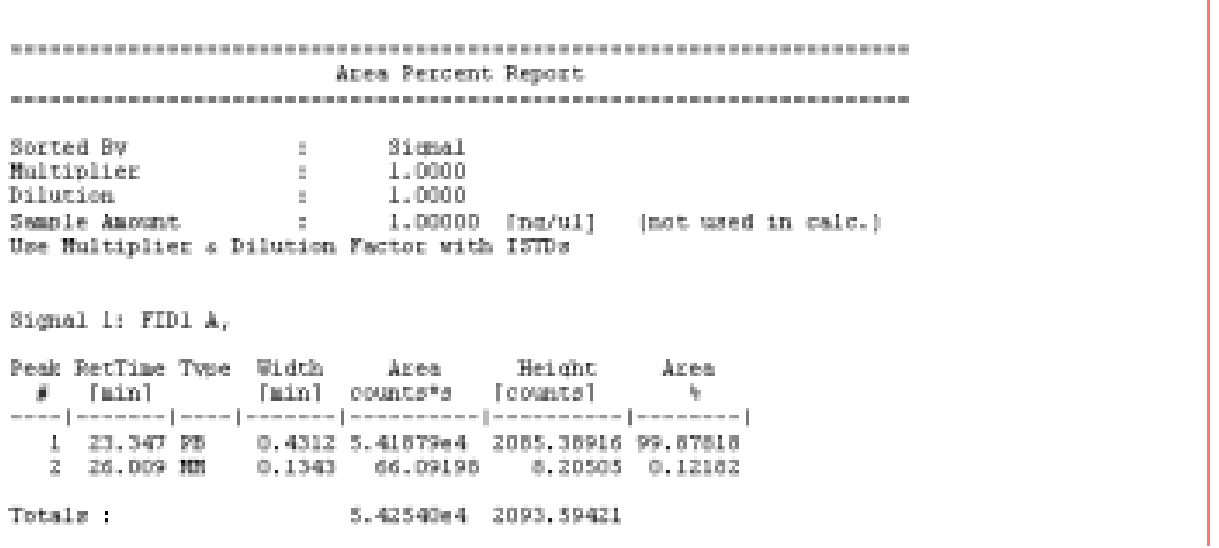

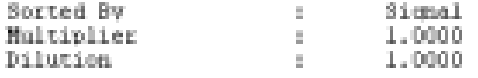

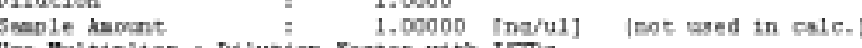

roctor with $15 \pi 0 a$

Signal 1: FIDI $d$,

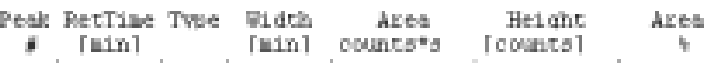

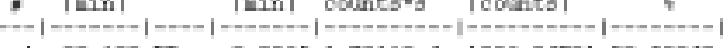

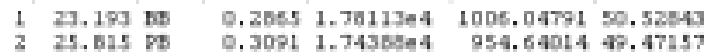

Tota1n ;

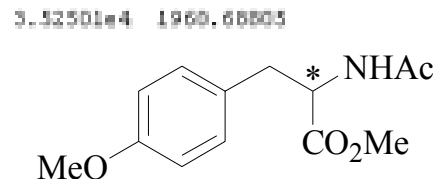

(racemic product)

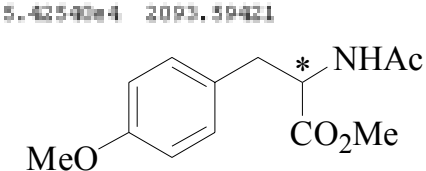

(hydrogenation product) 

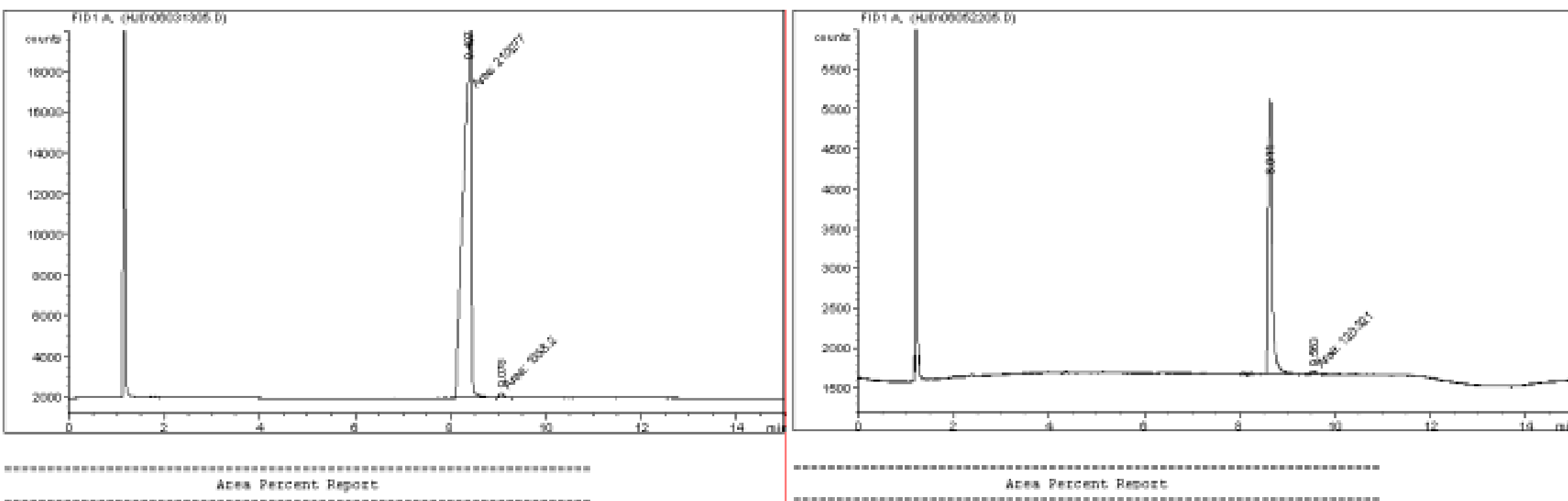

Ares Percent Regort

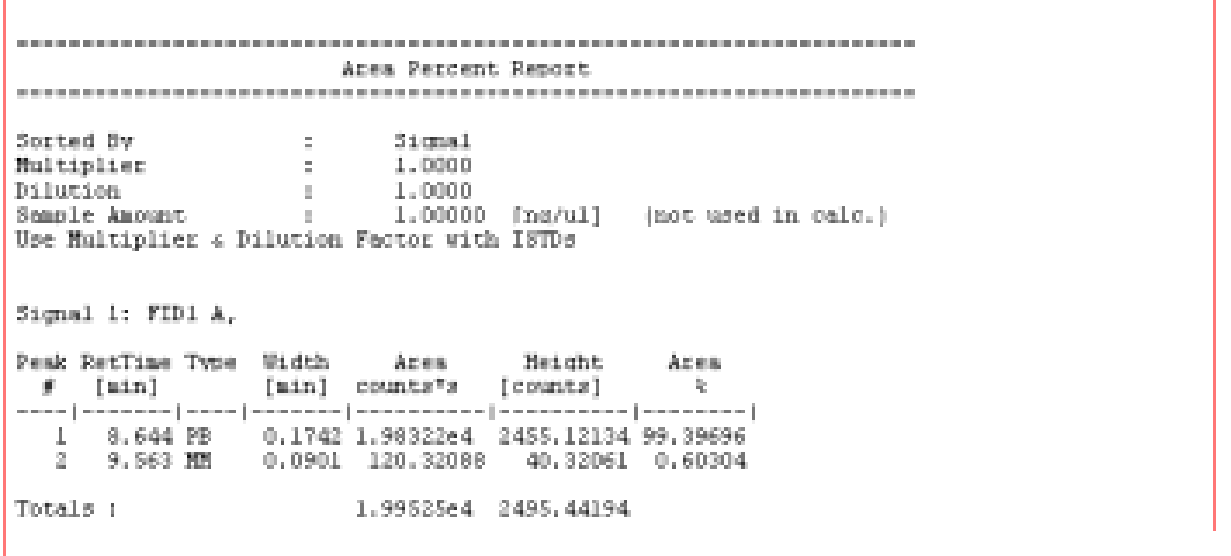

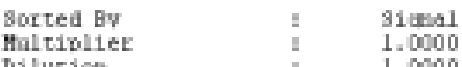

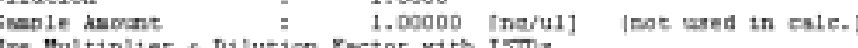

Signal 1: FID1 $d$,

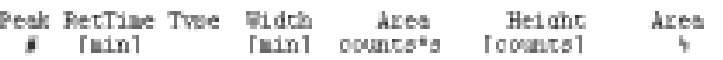

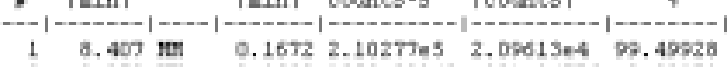

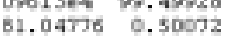

Totalz:

$2.1135595 \quad 2.1242404$

\section{* NHAc}

$\mathrm{CO}_{2} \mathrm{Me}$

(hydrogenation with

$0.02 \mathrm{~mol} \% \mathrm{Rh}$ )

Entry 12, Table 1

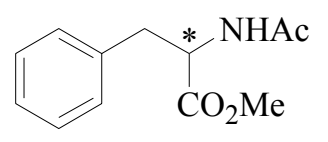

(hydrogenation with

$0.01 \mathrm{~mol} \% \mathrm{Rh}$ )

Entry 13 , Table 1 


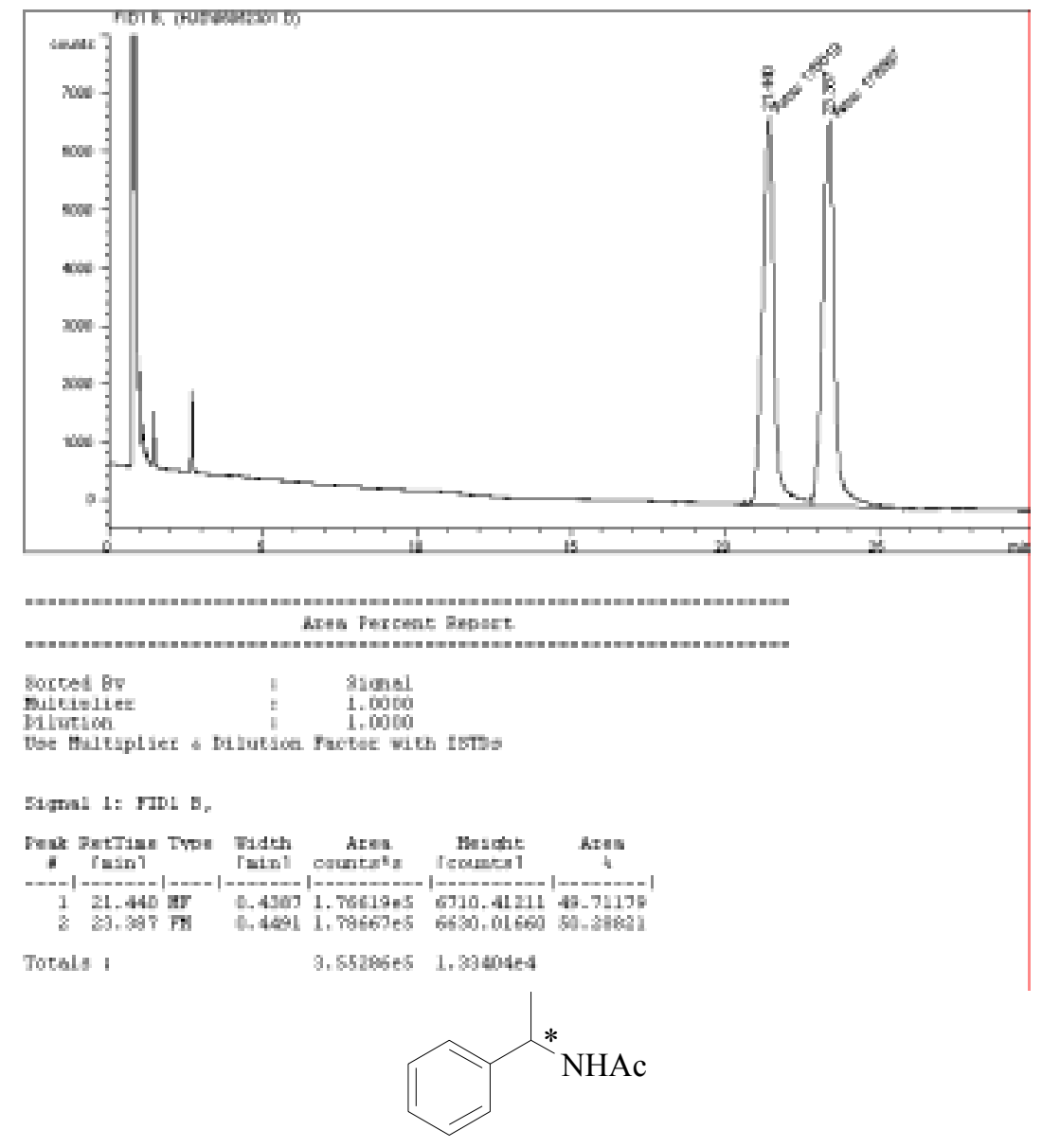

(racemic sample)

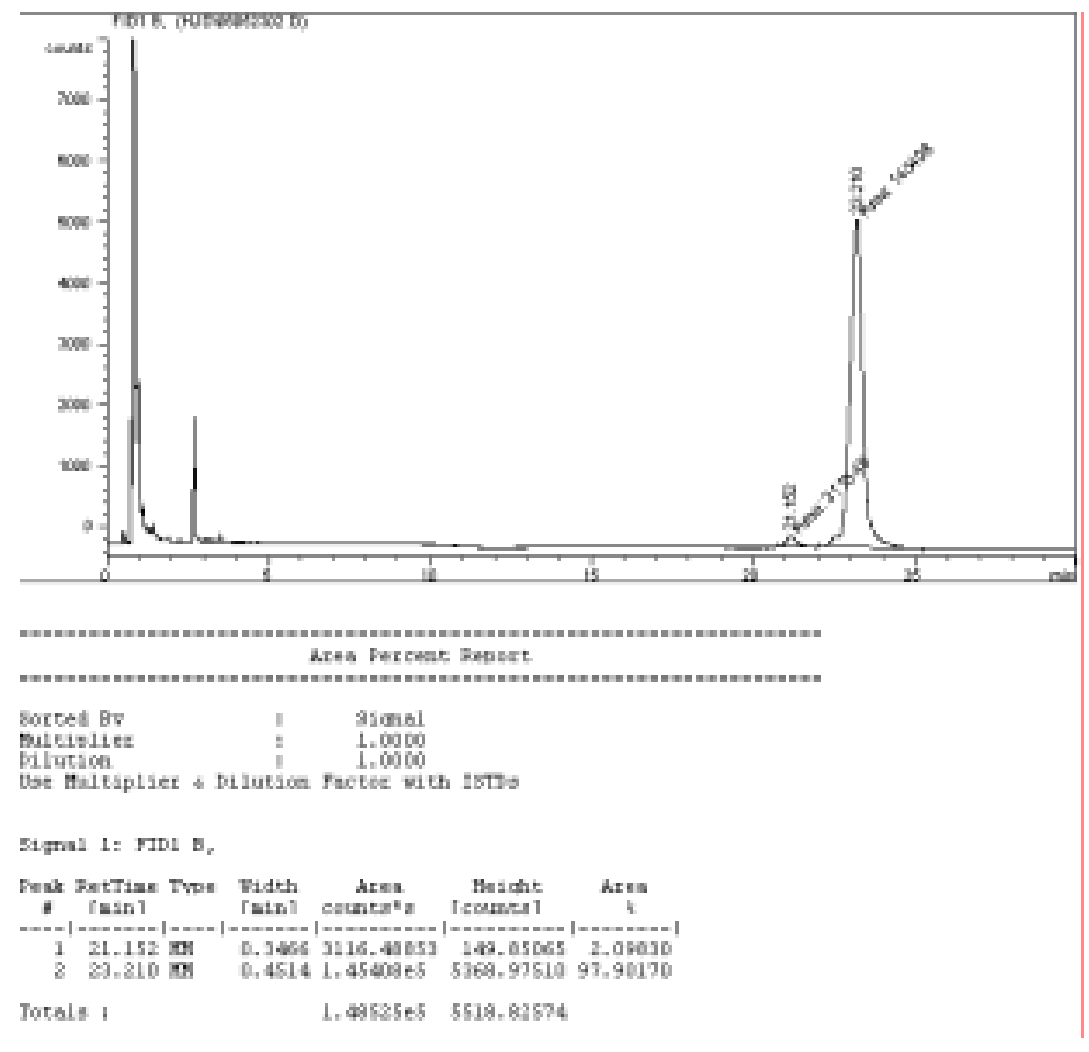

NHAc

(hydrogenation product

using PEAPhos-1a)

Entry 1, Table 2 


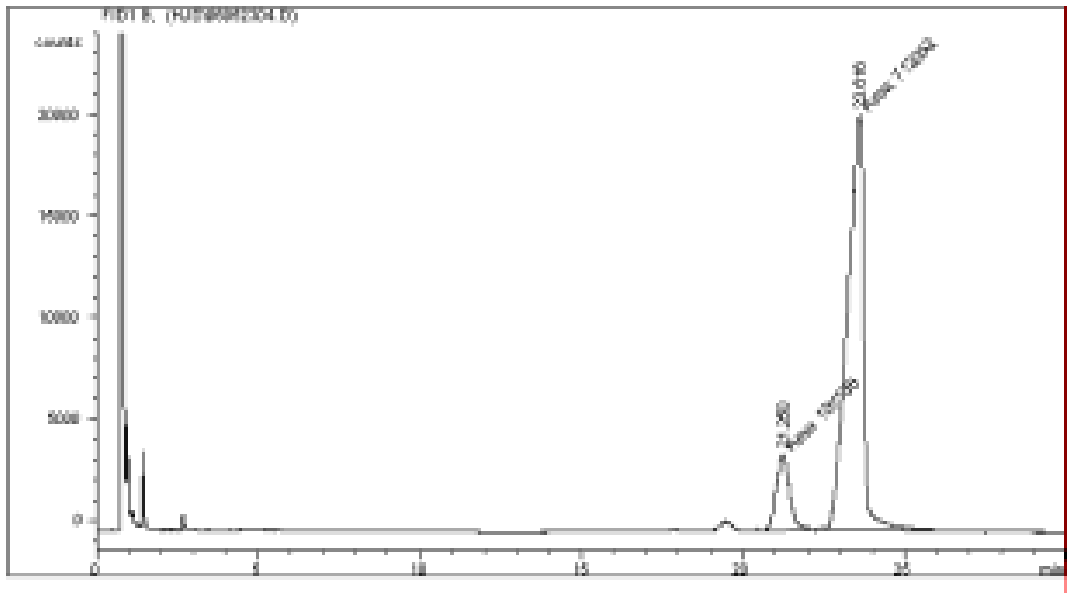

Ares Percent Bepart

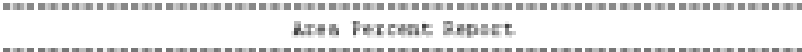

gocreet Bv Simes
$5 \quad 1.0000$
5

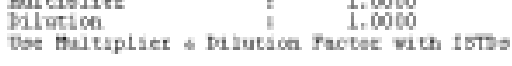

Stgmal 1: $\mathrm{MDD} \mathrm{B}$,

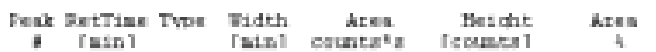

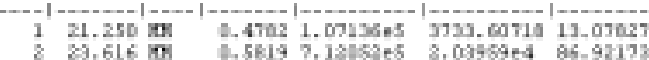

Totals । B. $19169 \mathrm{es}$ a alzased

* NHAc

(hydrogenation product

using PEAPhos-1b)

Entry 2, Table 2

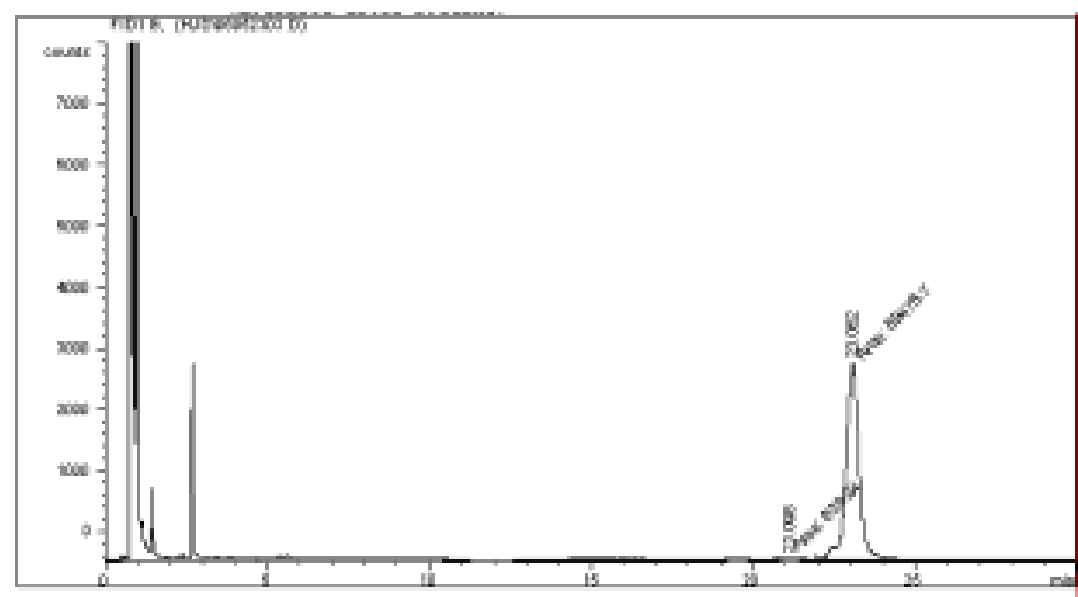

Ares Vercent Repart

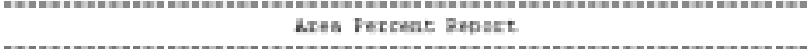

\section{Sorced Bv} 1.0060

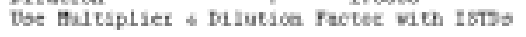

Sigmal 1: $\mathrm{TBD}$ is,

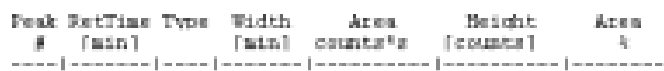

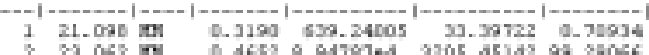

Totals

9.01179e4 3289.6466?

* NHAc

(hydrogenation product

using PEAPhos-1c)

Entry 3, Table 2 


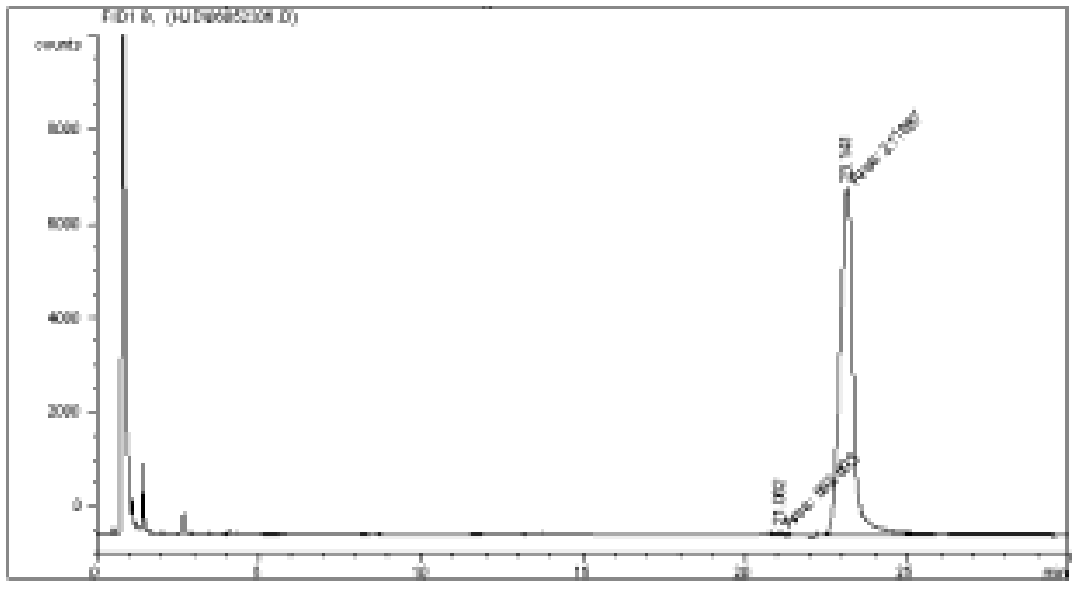

Aited Fetredt Wedort

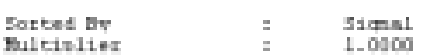

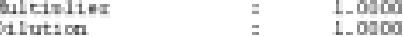

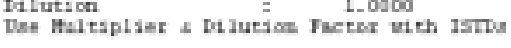

Simol L FTDI $B$,

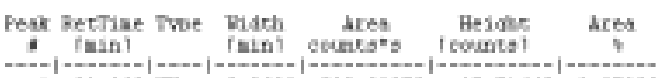

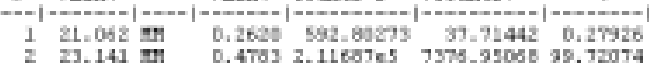

Totals,

2.12201es 7414-68510

NHAc

(hydrogenation product

using PEAPhos-1d)

Entry 4, Table 2 


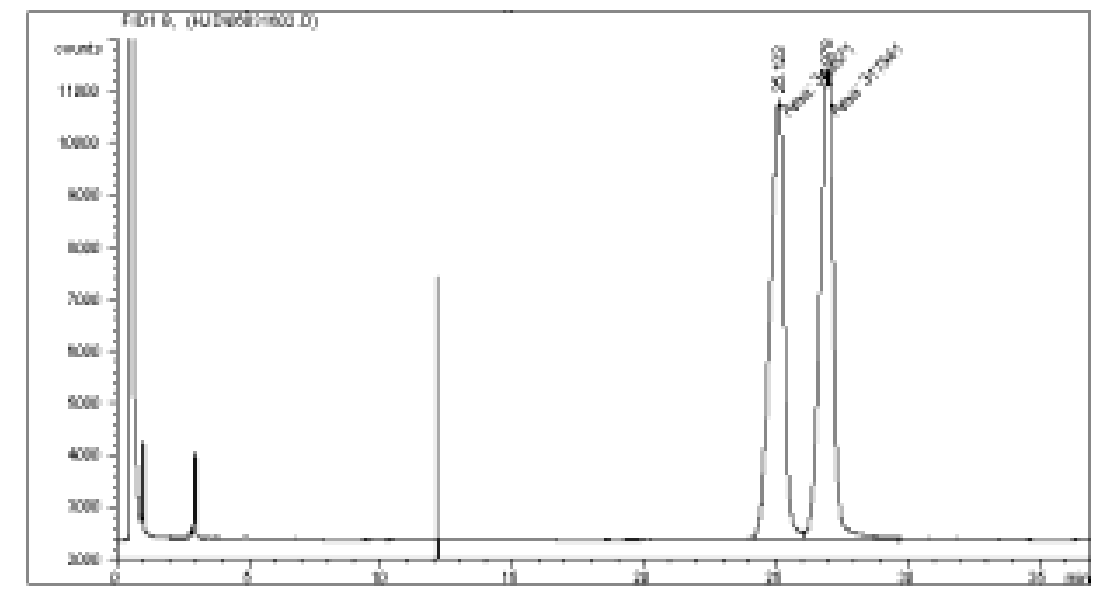

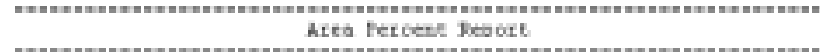

\section{Sorted Dv}

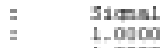

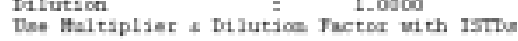

Simoil II FIDI B。

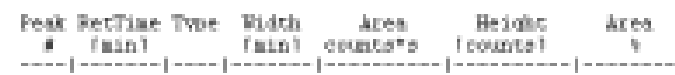

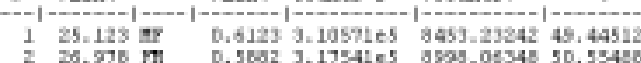

Totala :

6.23112es 1.74513e4

\section{$\mathrm{NHAc}$}

$\mathrm{Me}$

(racemic sample)

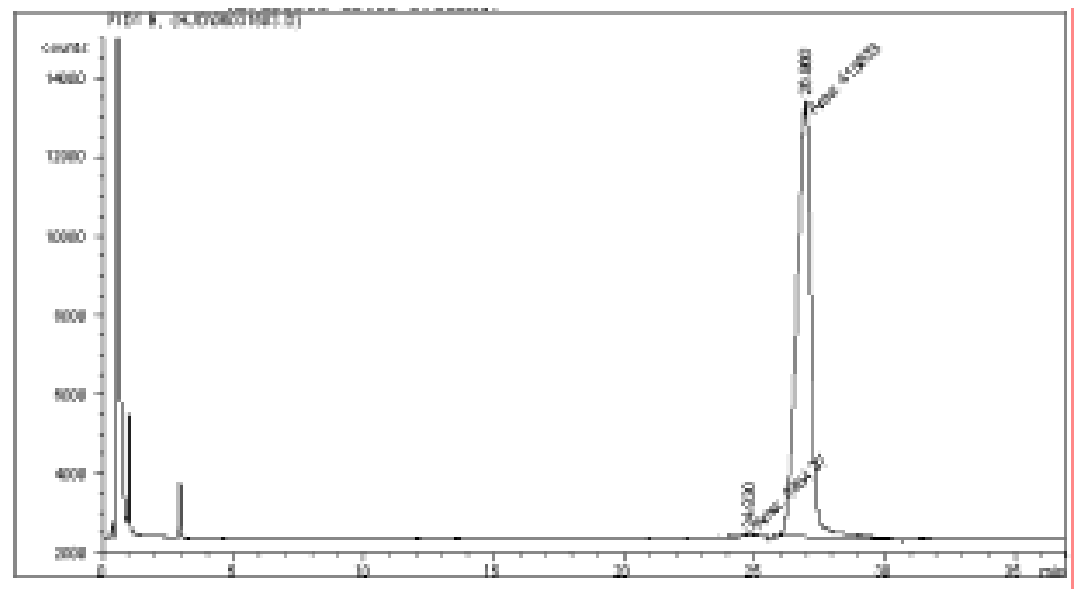

Ares Percen- Fenest

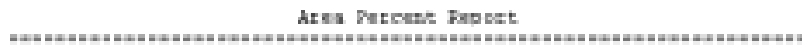

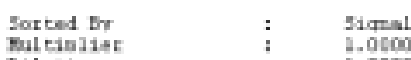

Dileveten

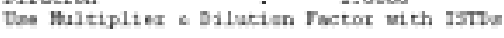

Signal 1: Trol $\mathrm{E}$.

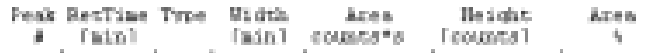

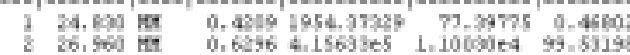

Totols 1

4.17897es 1.10604ed

\section{$\mathrm{NHAc}$}

$\mathrm{Me}$

(hydrogenation product)

Entry 5, Table 2 


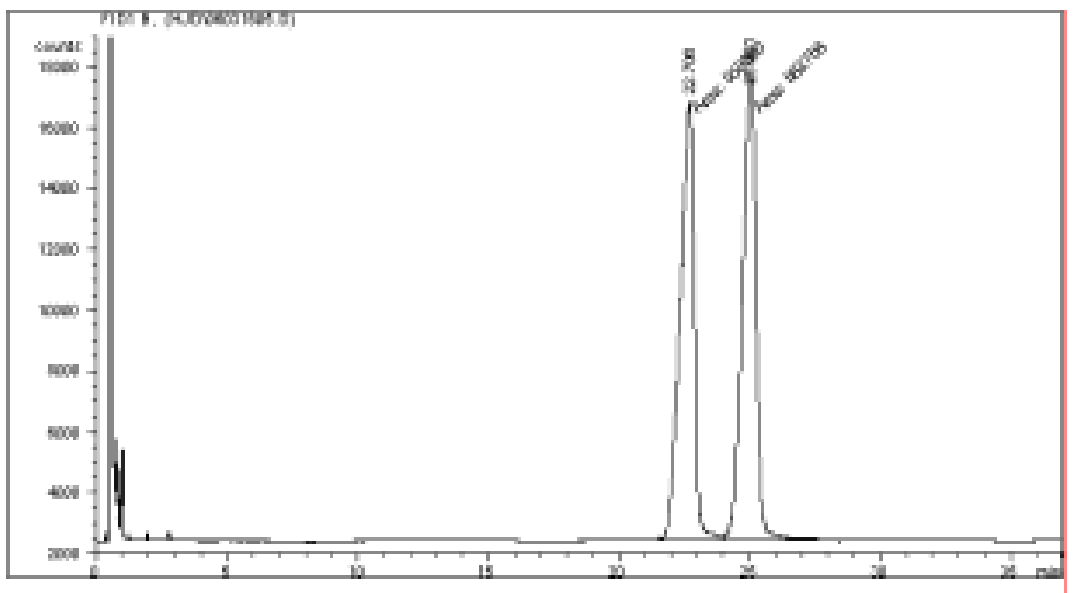

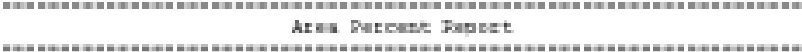

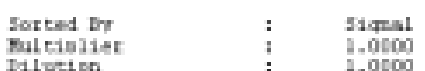

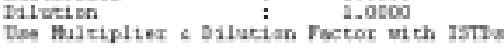

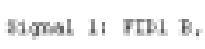

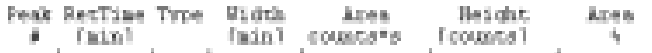

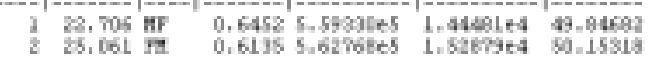

Totals ।

1.12atle 2.9nseoted

\section{NHAc}

$\mathrm{F}_{3} \mathrm{C}$

(racemic sample)

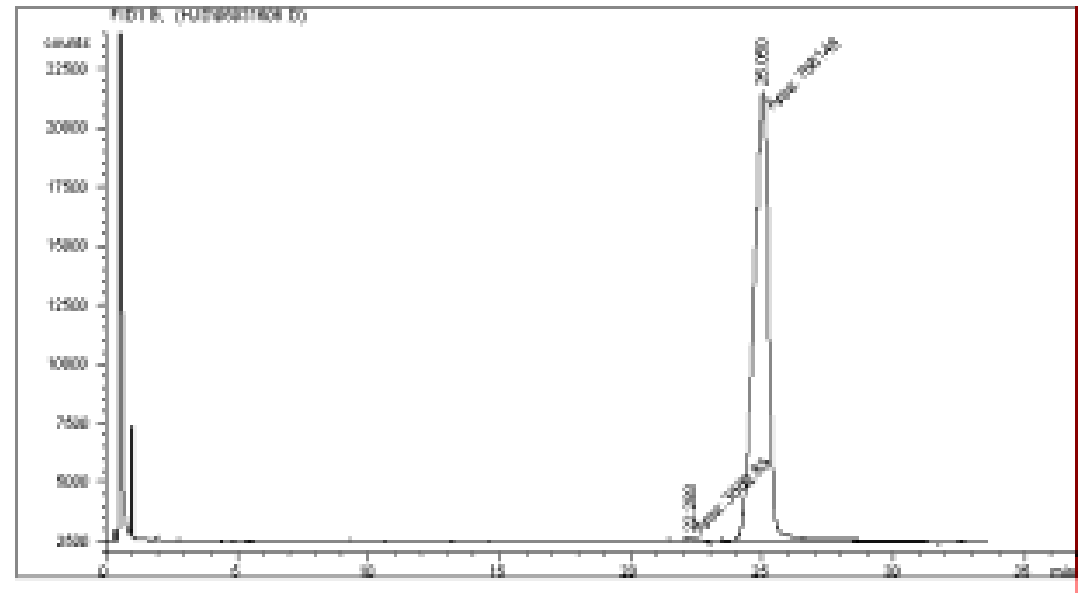

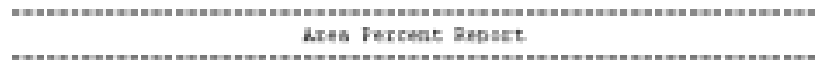

soroed iv

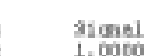

bilution

tos miltapliet o Dilution facter vich IsTs

Sigmal 1: $\mathrm{MDL}$ B,

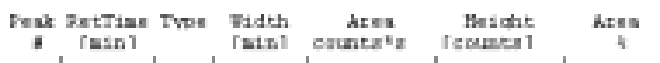

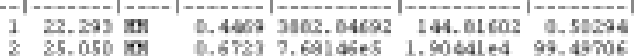

Totals !

7. rances 1.91690 ed

\section{NHAc}

$\mathrm{F}_{3} \mathrm{C}$

(hydrogenation product)

Entry 6, Table 2 

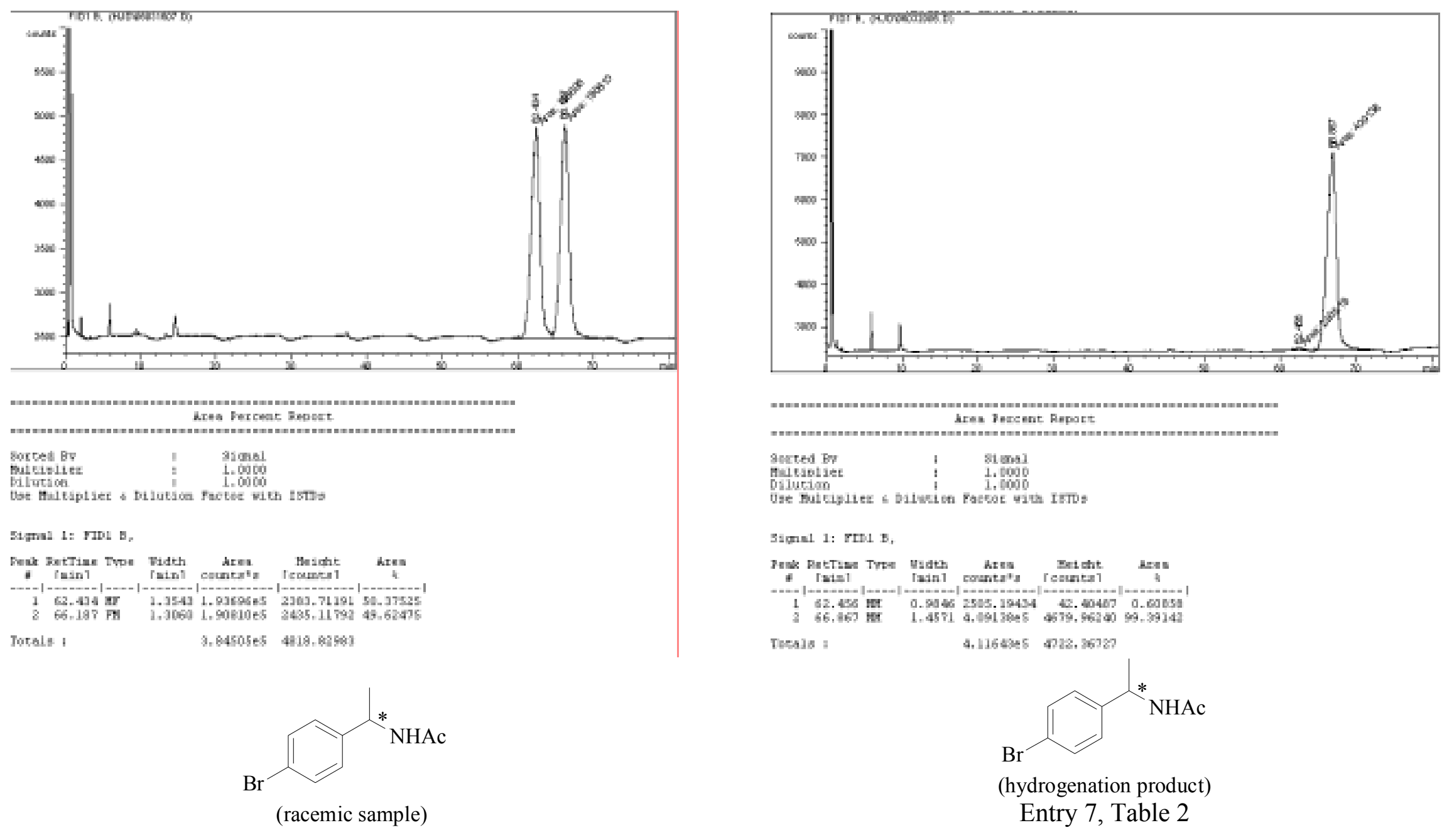

gected $\mathrm{BV}$

Fitesolie

simas

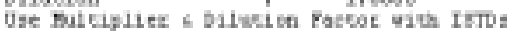

3squal 1: rrou s,

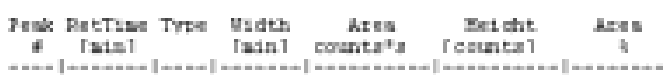

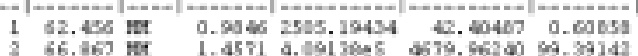

resals

4.116405 क12.3678?

* NHAc

$\mathrm{Br}$

(hydrogenation product)

Entry 7, Table 2 


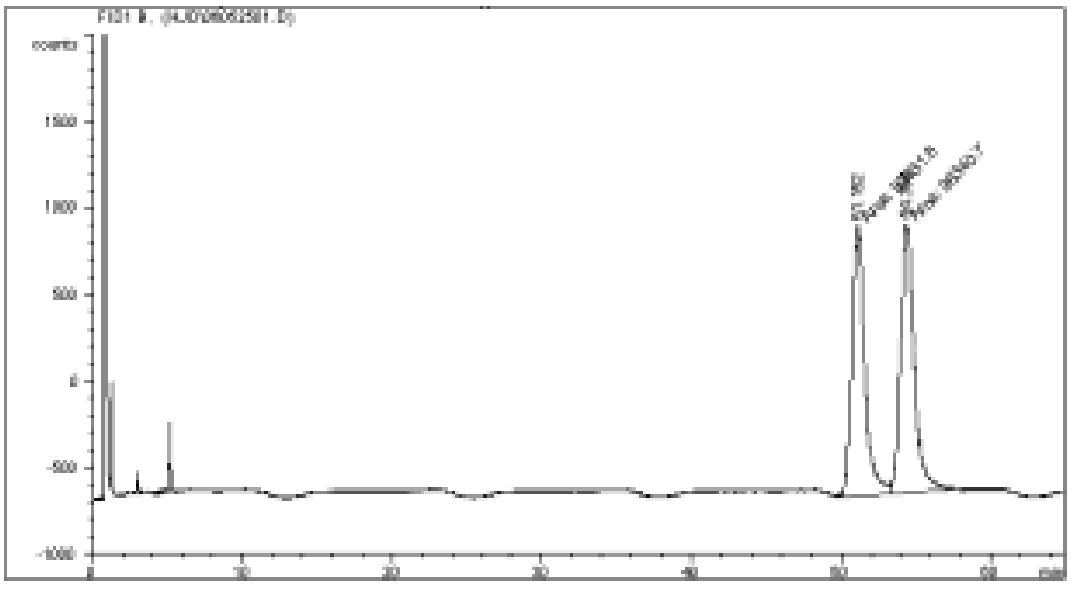

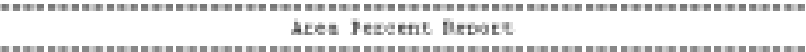

Sected Bv,

1. $\cos _{0}$

$1 . \cos 0$

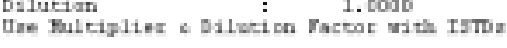

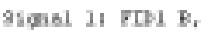

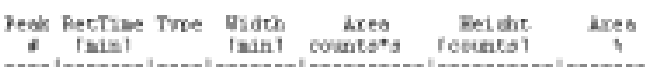

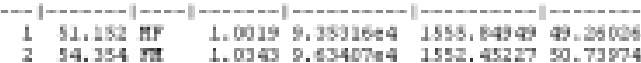

Totala = 1.39072as 3160.30178

\section{NHAc}

$\mathrm{Cl}$

(racemic sample)

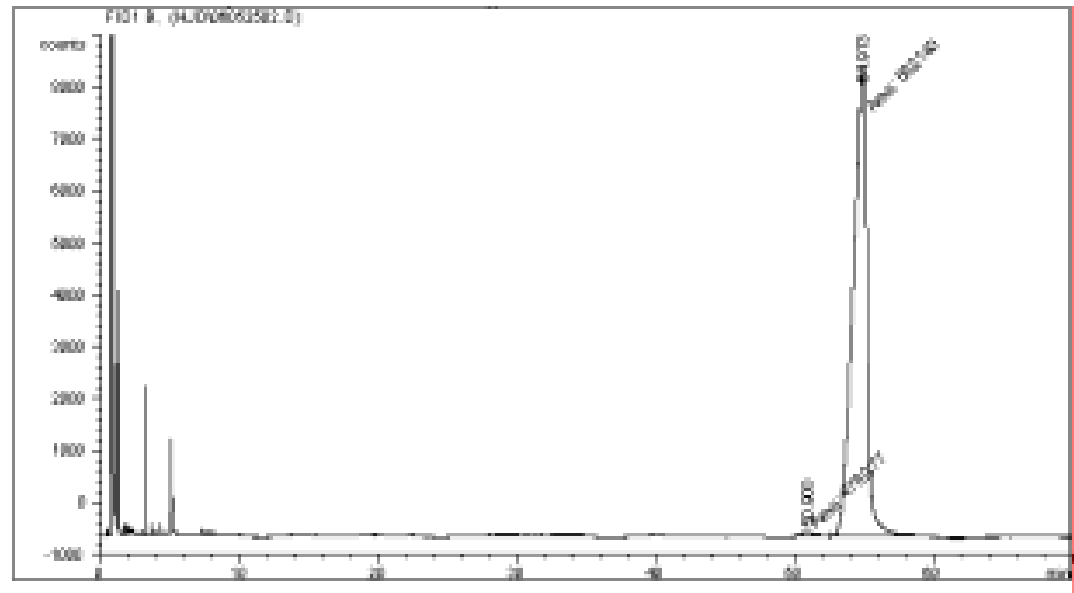

Ates Peteent hetort

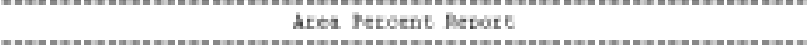

Serted DV

\section{$51 \mathrm{mal}$
1.0000
3.0000}

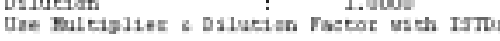

Signal if FTD $\mathrm{B}$,

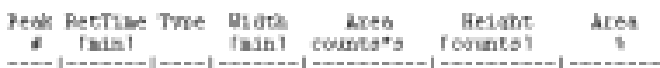

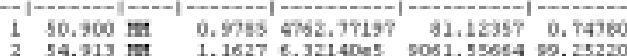

treala = e.semses 2142.68021

\section{NHAc}

$\mathrm{Cl}$

(hydrogenation product)

Entry 8 , Table 2 


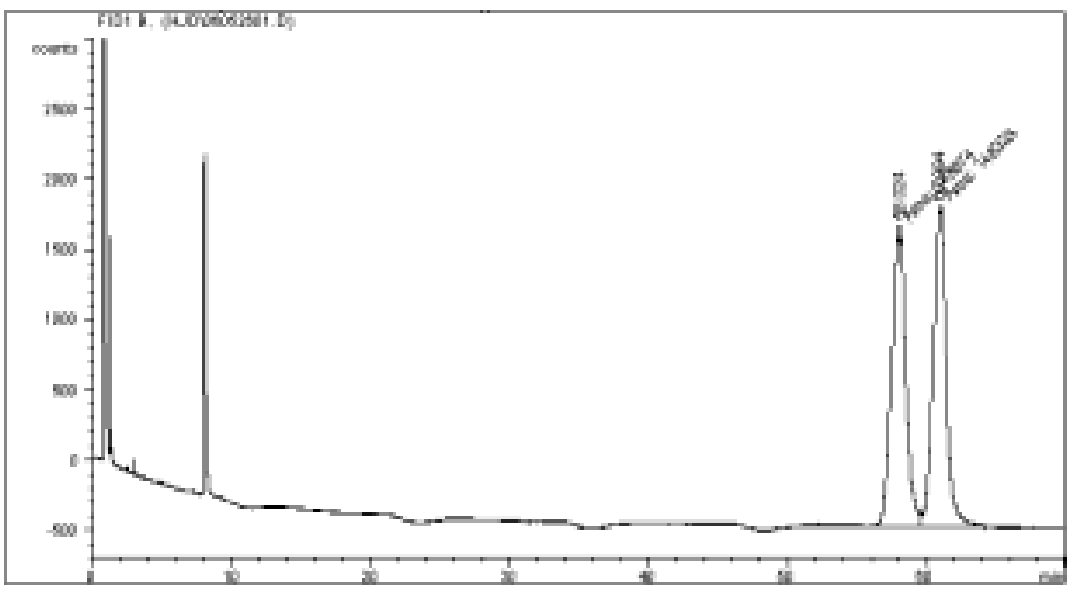

Ates Peacent Pevote

3ested $5 v$

1. $\cos _{0}$

1.

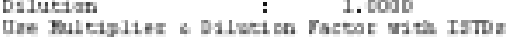

81.

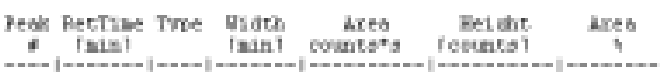

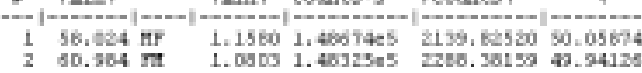

Treala :

2.3605005 4428.2005

NHAc

$\mathrm{MeO}$

(racemic sample)

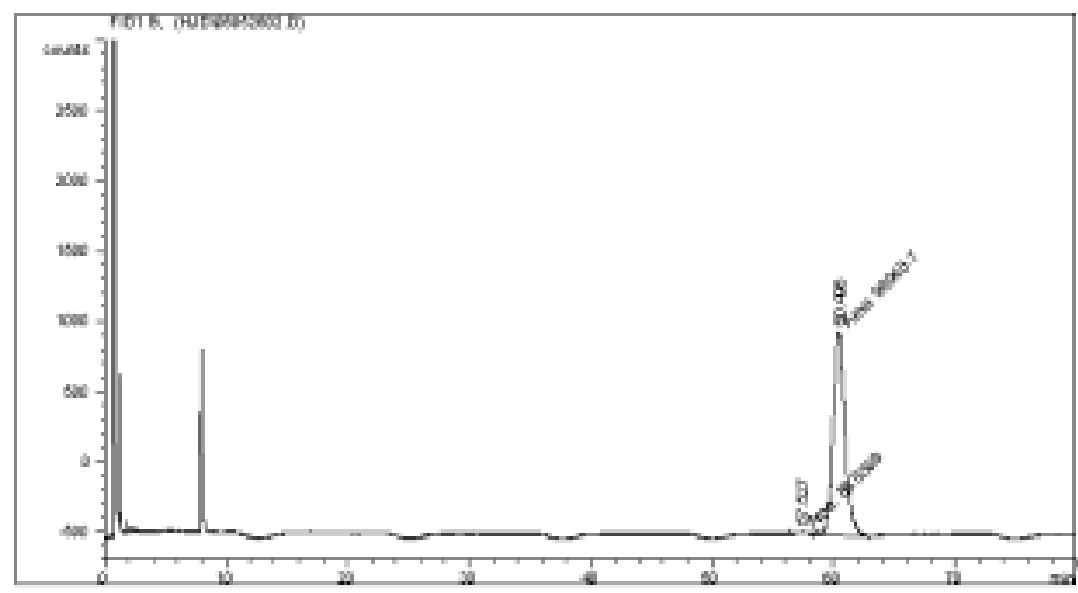

Axtea Dercens Besort

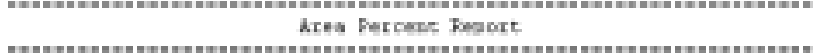

sorceet Bv

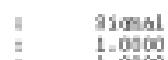

Bilution

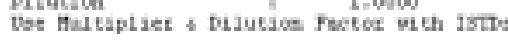

Stgmal 1: MDL B,

Deak BetTine Tore Width Ares Matat area

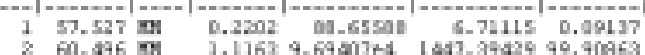

Totals !

9. 20297$) 4 \quad 1454.10544$

\section{NHAc}

$\mathrm{MeO}$

(hydrogenation product)

Entry 9, Table 2 


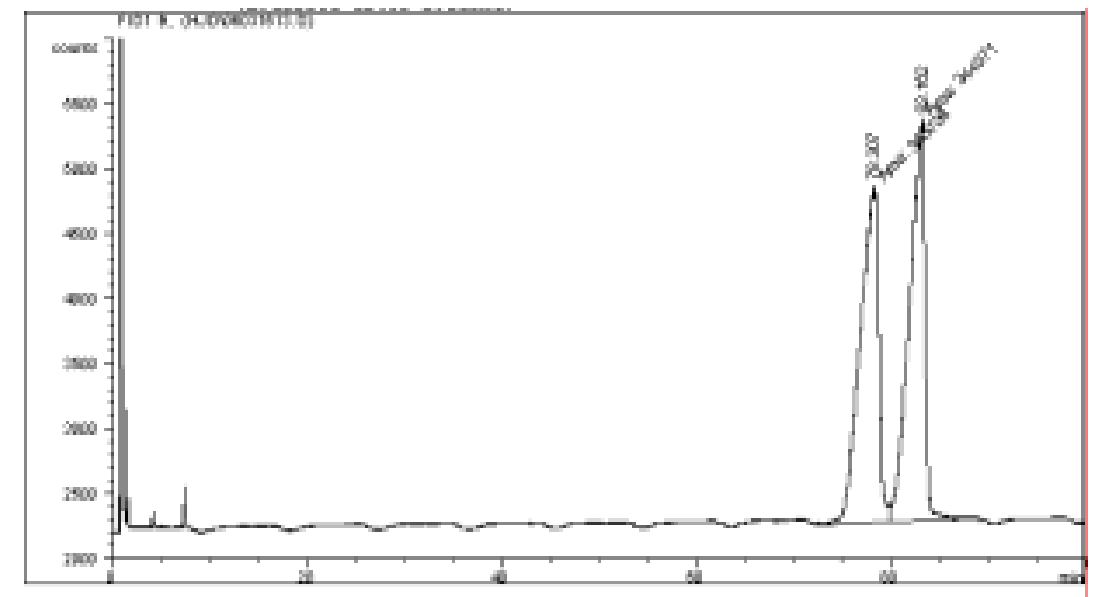

Ares pereent Rapost

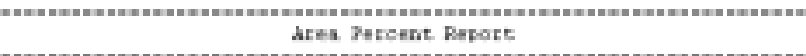

gected $\mathrm{kg}$

\section{$\operatorname{simal}_{1.0000}$}

Autsolie

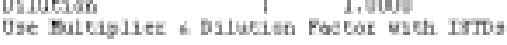

3smal 1: rmo s,

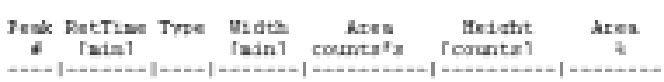

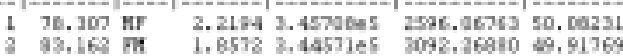

recass

$6.90879055698,3354$

\section{NHAc}

\section{$\mathrm{OMe}$}

(racemic sample)

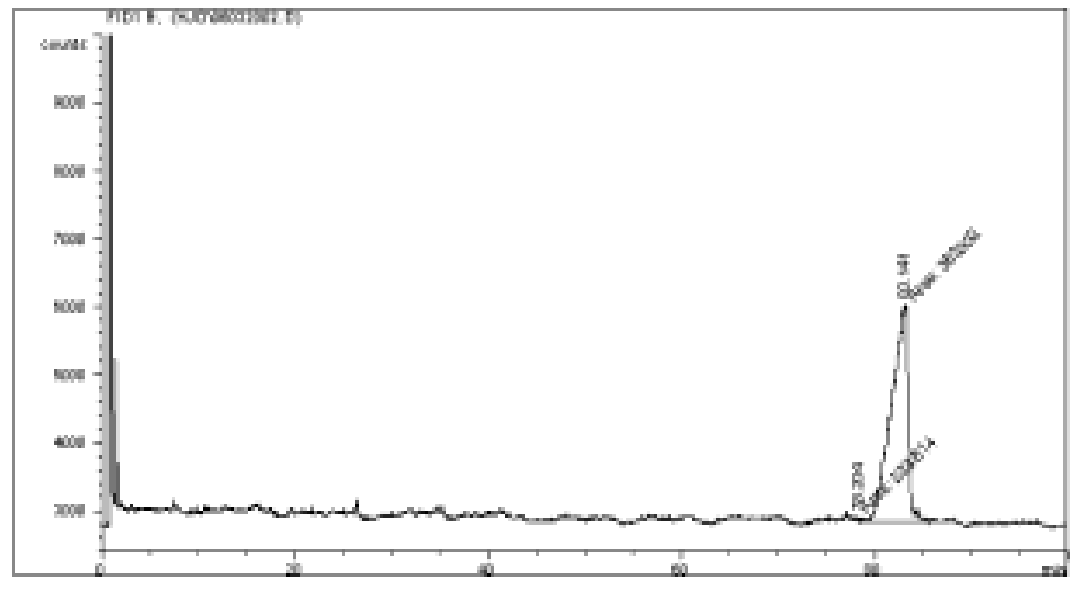

Ares Bacent Perote

Area hesectis Deroort

Sorced Dr

: $\quad 51$ mal

Ditution

simal it F"D B,

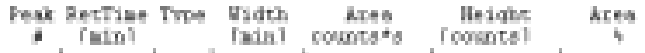

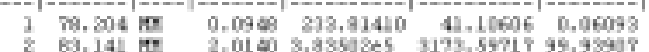

Totals ।

8.9303605 3814.3632

NHAc

$\mathrm{OMe}$

(hydrogenation product)

Entry10, Table 2 